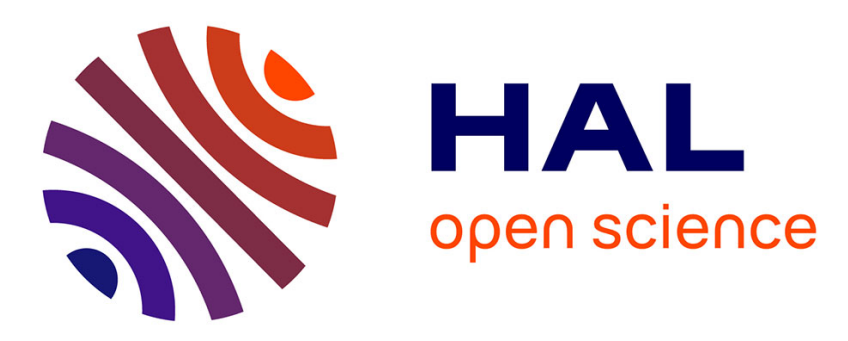

\title{
Identification of Heterogeneous Elastoplastic Behaviors Using the Constitutive Equation Gap Method
}

Tarik Madani, Yann Monerie, Stéphane Pagano, Céline Pelissou, Bertrand Wattrisse

\section{- To cite this version:}

Tarik Madani, Yann Monerie, Stéphane Pagano, Céline Pelissou, Bertrand Wattrisse. Identification of Heterogeneous Elastoplastic Behaviors Using the Constitutive Equation Gap Method. Experimental Mechanics, 2018, 58 (6), pp.919 - 939. 10.1007/s11340-018-0389-0 . hal-01898254

\section{HAL Id: hal-01898254 https://hal.science/hal-01898254}

Submitted on 18 Oct 2018

HAL is a multi-disciplinary open access archive for the deposit and dissemination of scientific research documents, whether they are published or not. The documents may come from teaching and research institutions in France or abroad, or from public or private research centers.
L'archive ouverte pluridisciplinaire HAL, est destinée au dépôt et à la diffusion de documents scientifiques de niveau recherche, publiés ou non, émanant des établissements d'enseignement et de recherche français ou étrangers, des laboratoires publics ou privés. 


\title{
Identification of Heterogeneous Elastoplastic Behaviors Using the Constitutive Equation Gap Method
}

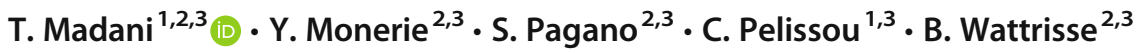

\begin{abstract}
Recent developments in imaging techniques now facilitate local field measurements (e.g. strain, temperature, etc.). In this study, we identify the spatial distribution of material properties and local stress fields using an inverse identification method based on the constitutive equation gap (CEG). The CEG concept is based both on minimization of a cost function equal to the sum of the potential and complementary energies, and on the deviation between the measured and computed strain fields. We propose a new approach for identifying heterogeneous property fields (mechanical parameters and stress) using a secant elastoplastic tensor and a measured strain field obtained by full-field measurement. The reliability of the method is checked using finite element simulation data as reference full-field measurements. The method is then applied on noisy displacement fields to assess its robustness. Finally, the developed inverse method is tested on real measured data.
\end{abstract}

Keywords Inverse method $\cdot$ Material identification $\cdot$ Elastoplasticity $\cdot$ Full field measurement

\section{Introduction}

Full-field kinematic measurements (digital image correlation, interferometric techniques, grid methods, etc.) [1] offer a very rich tool to gain insight into and characterize the micromechanical behavior of materials. Local displacement fields and overall loadings on the boundary allow identification of

\section{T. Madani}

tarik.madani@umontpellier.fr

Y. Monerie

yann.monerie@umontpellier.fr

S. Pagano

stephane.pagano@umontpellier.fr

C. Pelissou

celine.pelissou@irsn.fr

B. Wattrisse

bertrand.wattrisse@umontpellier.fr

1 IRSN/PSN-RES/SEMIA/LPTM, Institut de Radioprotection et de Sûreté Nucléaire, bât 702, BP3-13115, Saint-Paul-lez-Durance Cedex, France

2 LMGC, Université de Montpellier, CNRS, Montpellier, France

3 Laboratoire de micromécanique et intégrité des structures (MIST), IRSN-CNRS-Université de Montpellier, Montpellier, France constitutive law parameters via the resolution of an inverse problem [2-5].

The mechanical behavior identification techniques can be classified in five main categories (see [6] for an overview): the finite element model updating method (FEMU) [7-13], the reciprocity gap method (RGM) $[14,15]$, the constitutive equation gap method (CEGM) [16-18], the virtual field method (VFM) [19-27] and the equilibrium gap method (EGM) [28, 29].

We focus here on the CEGM to identify the constitutive law and its mechanical parameters for heterogeneous materials. This method - initially proposed by Ladeveze [30] to estimate errors in finite element simulations - was used by Constantinescu [31] and Geymonat [32] to identify elastic parameters and spatial stress distributions. Latourte [33] proposed an incremental version of the CEGM that introduces tangent and secant stiffness tensors to identify a distribution of elastoplastic fields. In the latter reference, the variational method is, however, limited to linear hardening behaviors and the stress field formulation (Airy functions) is only adapted to simple geometries and regular meshes. Furthermore, the two-step identification of plastic parameters (yield stress and hardening modulus) implies that the size of the plastic zone remains constant at every load step. The method proposed here intends to overcome these difficulties in order to describe both multilinear hardening behaviors of materials and complex geometries. 
The proposed CEGM is based on the minimization of an energy norm expressing the gap between a measured displacement field and a finite element computed one. This energy norm has to be understood in the sense of an elastoplastic secant stiffness tensor $\underline{\underline{B}} \underline{\underline{B}}$. This tensor is expressed as a function of both the elastic and plastic material properties and of the loading path. This tensor is equal to the standard elastic tensor $\underline{\underline{B}}^{e}$ for an elastic step (Hooke's law). The identification method proposed in this paper allows simultaneous identification of the entire set of plastic parameters.

Section 2 presents the CEGM framework where we propose a general presentation of the identification method and we describe the elastic and plastic identification principles. In section 3 , we detail the identification procedure and its numerical implementation. In section 4, the efficiency of the proposed approach is examined using reference measurements numerically obtained with the COMSOL Multiphysics finite element code. In the last section, the performance of the method in identifying material property fields is illustrated with experimental data.

\section{Constitutive Equation Gap Method}

Optical measurement techniques applied to full-field measurements are becoming increasingly popular in research laboratories because of the density of information they provide. The development and application of identification methods based on full-field measurements is an active research field that capitalizes on the large amount of data produced by imaging techniques. This study focuses on the identification of elastoplastic models. Hereafter, we describe the general principles of CEGM and then introduce the general concepts chosen to describe elastoplastic behavior.

\section{General Presentation of CEGM}

The procedure is presented within a $2 \mathrm{D}$ framework (plane stress) since the applications addressed here are related to thin flat samples. Only three in-plane strain components were thus investigated and only three material parameters were locally (i.e. on a given material domain) identified using a single displacement field. By taking several time steps into account, it was possible to increase the number of parameters that could be identified in each material domain.

The CEGM uses a statically admissible stress field $\sigma_{n}^{c}$ involving a secant stiffness tensor $B_{n}^{s}$ determined at each load step $n$ :

$\underline{\sigma_{n}^{c}}=\underline{\underline{B_{n}^{S}}}: \underline{\varepsilon_{n}^{c}}$ where the strain tensor $\underline{\varepsilon_{n}^{c}}$ is expressed as:

$\underline{\varepsilon_{n}^{c}}=\underline{\varepsilon}\left(\overrightarrow{u_{n}^{c}}\right)$

with $\underline{\varepsilon}()$ standing for the small strain operator and $\overrightarrow{u_{n}^{c}}$ representing the displacement field. We use the classical vector representation of the stress and strain tensors (Voigt's notation) such that the fourth order elastic and elastoplastic tensors are represented by symmetric matrices.

We considered a quasi-static problem over a domain $\Omega$ with stress-free boundaries $\partial \Omega_{i}$, boundaries $\partial \Omega_{j}$ loaded with overall known forces $\vec{R}_{j}$ and boundaries with prescribed displacements $\partial \Omega_{u}$ satisfying $\partial \Omega_{j} \cup \partial \Omega_{i} \cup \partial \Omega_{u}=\partial \Omega, \partial \Omega_{j} \cap \partial \Omega_{i}=\varnothing$, $\partial \Omega_{i} \cap \partial \Omega_{u}=\varnothing$ and $\partial \Omega_{j} \cap \partial \Omega_{u}=\varnothing$ :

$\left\{\begin{array}{l}\operatorname{div} \sigma_{n}^{c}=\overrightarrow{0} \text { in } \Omega \\ \vec{R}_{j}=\int_{\partial \Omega_{j}} \sigma_{n}^{c} \vec{n} d s \quad \text { on } \partial \Omega_{j} \\ \sigma_{n}^{c} \vec{n}=\overrightarrow{0} \text { on } \partial \Omega_{i} \\ \overrightarrow{\overrightarrow{u_{n}^{c}}}=\overrightarrow{u_{n}^{m}} \text { on } \partial \Omega_{u}\end{array}\right.$

where $\overrightarrow{u_{n}^{m}}$ stands for the measured displacement at load step $n$ and $\vec{n}$ is the outer unit vector.

For a sequence of $N$ successive load steps, the CEG functional $\left(F_{C E G M}\right)$ depends on two sets of parameters, i.e. the displacement field $\overrightarrow{u_{n}^{c}}$ and the mechanical material parameters on which $B_{n}^{s}$ depends, and reads:

$F_{C E G M}\left(\overrightarrow{u_{n}^{c}} B_{\underline{n}}^{s}\right)=\sum_{n=1}^{N} \int_{\Omega}\left[\underline{\varepsilon}\left(\overrightarrow{u_{n}^{c}}\right)-\underline{\underline{\varepsilon}}\left(\overrightarrow{u_{n}^{m}}\right)\right]: B_{\underline{n}}^{s}:\left[\underline{\varepsilon}\left(\overrightarrow{u_{n}^{c}}\right)-\underline{\varepsilon}\left(\overrightarrow{u_{n}^{m}}\right)\right] d \Omega$

Note that for a heterogeneous material the secant tensor depends on both the load step $n$ and the spatial position.

This procedure is applicable to any identification problem in which the secant tensor can be analytically expressed as a function of the material parameters and the loading state. Depending on the material behavior, the secant tensor can take different forms. In the next two sections, we describe the expressions of this tensor in the case of pure elasticity and elastoplasticity.

\section{Description of the Secant Tensor: Case of Cubic Elasticity}

In the case of cubic elasticity with a plane stress hypothesis, the standard Hooke stiffness tensor can be written as:

$\stackrel{B^{e}}{=}=\left[\begin{array}{ccc}b_{1} & b_{2} & 0 \\ b_{2} & b_{1} & 0 \\ 0 & 0 & b_{3}\end{array}\right]$ 
with $b_{1}={ }^{E} /{ }_{1-\nu^{2}}, b_{2}=v b_{1}$ and $b_{3}=2 G$. The quantities $E, v$ and $G$ are the Young modulus, the Poisson ratio and the shear modulus, respectively. This tensor depends only on three material parameters $\left(b_{1}, b_{2}\right.$ and $\left.b_{3}\right)$ that have to be determined using equation (4).

\section{Description of the Secant Tensor: Case of Prager Elastoplasticity}

The Prager linear kinematic hardening model formulated under the plane stress assumption involves two parameters: a yield stress $\sigma_{0}$ and a hardening modulus $h$. The corresponding behavior reads (implicit scheme and notations from [34]):

$\underline{\sigma_{n}^{c}}=\underline{\underline{B}}^{e}:\left(\underline{\varepsilon_{n}-\varepsilon_{\underline{n}}^{p}}\right)$

with a yield function $f$ depending on the back-stress tensor $X_{n}$ at step $n$ :

$f\left(\underline{\sigma_{n}^{c}}, \underline{X_{n}}\right)=\left(\underline{\sigma_{n}^{c}}-\underline{X_{n}}\right)_{e q}-\sigma_{0}=\sqrt{\underline{\eta_{n}{ }^{T}}: \underline{\underline{P}}: \underline{\eta_{n}}}-\sqrt{\frac{2}{3}} \sigma_{0} \leq 0$

where subscript ()$_{e q}$ stands for the von Mises equivalent stress, and:

$\underline{\underline{P}}=\frac{1}{3}\left[\begin{array}{ccc}2 & -1 & 0 \\ -1 & 2 & 0 \\ 0 & 0 & 6\end{array}\right]$ and $\underline{\eta_{n}}=\sigma_{n}^{c}-\underline{X_{n}}$

The incremental flow rule reads:

$\underline{\Delta \varepsilon_{n}^{p}}=\Delta \gamma_{n} \frac{\partial f}{\partial \sigma_{n}^{c}}=\Delta \gamma_{n} \underline{P}: \underline{\eta_{n}}$

where $\Delta \gamma_{n}$ represents the plastic multiplier at load step $n$.
Finally, the back-stress increment $\Delta X_{n}$ reads:

$\underline{\Delta X_{n}}=\frac{2}{3} h \Delta \gamma_{n} \underline{\eta_{n}}$

From these equations, we deduced the expression of $\sigma_{n}^{c}$ by:

$\underline{\sigma_{n}^{c}}=\left[\underline{\underline{B}}^{e-1}+\frac{\Delta \gamma_{n}}{1+\frac{2}{3} h \Delta \gamma_{n}} \underline{P}\right]^{-1}:\left[\left(\underline{\varepsilon}_{\underline{n}}^{c}-\underline{\varepsilon_{n}^{p}}\right)+\frac{\Delta \gamma_{n}}{1+\frac{2}{3} h \Delta \gamma_{n}} \underline{P}: \underline{X_{n}}\right]$

Equation (11) is established for any situation where the initial state $\left(X_{n}\right.$ and $\left.\underline{\varepsilon}_{n}^{p}\right)$ and the plastic increment $\left(\Delta \gamma_{n}\right)$ are known. In our situation, we adopt a secant formulation for plasticity and we assume that the initial state corresponds to vanishing plastic deformations and back-stress. The incremental plastic strains and back-stresses become significant when plasticity develops. Their variations are governed by equations (9) and (10).

For a plastic step with no initial hardening (i.e. $X_{n}=0$ and $\varepsilon_{\underline{n}}^{p}=0$ ), an explicit formula for the elastoplastic secant tensor $\underline{\underline{B_{n}^{s}}}$ was obtained:

$\underline{\sigma_{n}^{c}}=\underline{\underline{B_{n}^{s}}}: \underline{\varepsilon_{n}^{c}}$

where

$\underline{\underline{B_{n}^{s}}}=\left[\underline{\underline{B^{e-1}}}+\frac{\Delta \gamma_{n}}{1+\frac{2}{3} h \Delta \gamma_{n}} \underline{\underline{P}}\right]^{-1}$

This tensor $B_{n \_}^{s}$ could be expressed with respect to the elastic parameters $(E, G$ and $\nu)$ and plastic parameter $K_{n}$ at load step $n$ :

$$
\underline{\underline{B_{n}^{s}}}=\left[\begin{array}{ccc}
\frac{E\left(1+2 K_{n} E\right)}{3 K_{n}^{2} E 2-2 K_{n} E(\nu-2)+1-\nu 2} & \frac{E\left(\nu+K_{n} E\right)}{3 K_{n}^{2} E 2-2 K_{n} E(\nu-2)+1-\nu 2} & 0 \\
\frac{E\left(\nu+K_{n} E\right)}{3 K_{n}^{2} E 2-2 K_{n} E(\nu-2)+1-\nu 2} & \frac{E\left(1+2 K_{n} E\right)}{3 K_{n}^{2} E 2-2 K_{n} E(\nu-2)+1-\nu 2} & 0 \\
0 & 0 & \frac{2 G}{1+12 K_{n} G}
\end{array}\right]
$$

with $K_{n}$ defined as:

$K_{n}=\frac{\Delta \gamma_{n}\left(\sigma_{0}\right)}{3+h \Delta \gamma_{n}\left(\sigma_{0}\right)}$

The plastic parameter $K_{n}$ depends both on the plastic material parameters (the yield stress $\sigma_{0}$ and the hardening modulus $h$ ) and on the local mechanical loading (through $\left.\Delta \gamma_{n}\right)$. Note that the secant tensor is equal to the elastic tensor when the plasticity does not evolve, i.e. when $K_{n}=0$.
Furthermore, both tensors have always the same structure (even when plasticity occurs) as they are symmetric with two equal components " 11 " and " 22 " and vanishing components " 13 " and " 23 " (in the matrix representation).

Substituting the expression of $\Delta \gamma_{n}\left(\sigma_{0}\right)$, we have:

$$
K_{n}=\frac{1}{2 h}\left(1-\sqrt{\frac{2}{3}} \sigma_{0} / \alpha_{n}\right)
$$


where the quantity $\alpha_{n}$ is given by:

$\alpha_{n}=\sqrt{\left(\sigma_{n^{-}}^{c}\right)^{T}: \underline{\underline{P}}:\left(\underline{\sigma_{n}^{c}}\right)}$

It is possible to rewrite the plastic parameter $K_{n}$ as a function of the norm of the equivalent plastic deformation $\left\|\varepsilon_{n}^{p}\right\|$ which is easily accessible in the Finite Element description and as a function of two independent plastic parameters $a_{K}$ and $b_{K}$ (depending only on $\sigma_{0}$ and $h$ ):

$K_{n}=a_{K} * \frac{\left\|\varepsilon_{n}^{p}\right\|}{b_{K}+\left\|\varepsilon_{n}^{p}\right\|}$ with $a_{K}=\frac{1}{2 h}$ and $b_{K}=\frac{\sigma_{0}}{h}$

with $\left\|\underline{\varepsilon_{n}^{p}}\right\|$ equal to:

$\left\|\underline{\varepsilon_{n}^{p}}\right\|=\sqrt{\frac{2}{3}\left(\varepsilon_{\underline{x x}}^{p^{2}}+\varepsilon_{\underline{y y}}^{p^{2}}+\left(\underline{\varepsilon_{x x}^{p}}+\underline{\varepsilon_{y y}^{p}}\right)^{2}+2 \underline{\varepsilon_{x y}^{p 2}}\right)}$

Expressions (1.16) to (1.18) of the elastoplastic secant tensor $\underline{B}_{n}^{S}$ show that it is not possible to simultaneously identify the couple $\left(h, \sigma_{0}\right)$ using a single load step for a homogeneous test. The identification of these quantities requires either two load steps for a homogeneous stress field, or a single load test for a non- homogeneous stress field.

\section{Minimization of the CEG Functional}

In this section, the computation and minimization of the CEG cost function are detailed. The convexity property of the cost function was proved in the elastic case by Geymonat [23]. Since the secant tensor has the shape of a cubic elastic tensor, this property extends to the elastoplastic case as soon as the secant tensor remains positive-definite. This property allows the use of a relaxation method for successively minimizing the functional with respect to its first argument (i.e. the displacement field $\overrightarrow{u_{n}^{c}}$ associated with the stress field $\underline{\sigma_{n}^{c}}$ ) and then to its second argument (i.e. the material properties introduced in the expression of the secant tensor $B_{n}^{s}$ ).

The separate convexity property ensures the existence and uniqueness of the global minimum of the optimization problem and the existence of a unique solution for each minimization. These minimizations were performed within the finite element framework. The elastic and plastic properties were consecutively identified. The developed algorithm consists in:

$1 /$ identifying the elastic properties,

2 / determining the time step at which the plasticity locally occurs,
3/ identifying the plastic properties starting from this plasticity onset.

These three steps are described hereafter.

\section{Spatial Discretization of the Fields Involved in the Identification}

As already noted, three fields have to be defined in order to compute the CEG functional: the phase distribution (accounting for the mechanical property heterogeneity), the stress fields, and the experimental displacement fields obtained by DIC. These three fields are discretized on different meshes with adapted shape functions. The corresponding meshes are 'nested' one into each other: the 'DIC mesh' is a subdivision of the 'stress mesh', which is itself built as a subdivision of the 'mechanical properties mesh' (see Fig. 1). The shape functions used to describe the fields on these three meshes are different. The stresses are determined via a FE computation using bilinear displacement elements. The local DIC computation is here performed using bilinear shape functions (higher order descriptions are available). The continuity of the displacement field is enforced by averaging the displacement on the mesh vertexes. Finally, the mechanical properties are constant on each material domain.

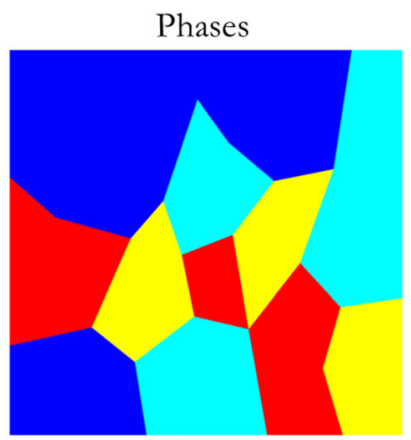

(a)

Plastic identification

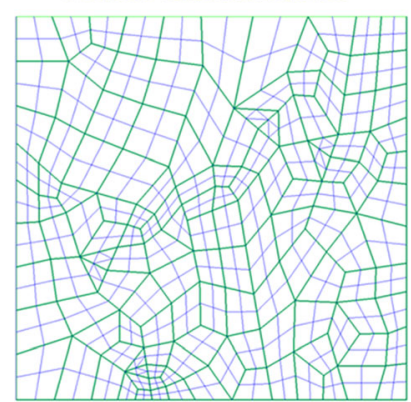

(c)

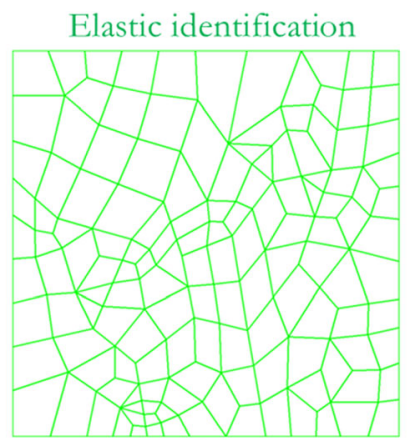

(b)

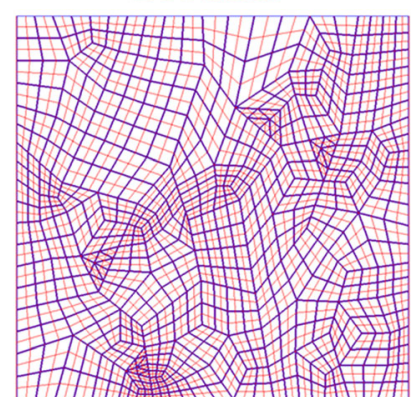

(d)

Fig. 1 Example of meshes used to describe: (a) the material properties (here 4 physical domains associated with 4 phases) (b) the elastic stress field (138 elements) (c) the plastic stress field (552 elements) and (d) the DIC-measured displacement field (2208 elements) 
Moreover, it is possible to use different stress meshes in both elasticity and plasticity. We assume that stress gradients can be higher in plasticity than in elasticity due to possible plastic localization. The 'plastic mesh' is thus a subdivision of the 'elastic mesh' in order to reduce the influence of noise on the identification, while maintaining a convenient description of the stress gradients. To conclude, four meshes were introduced, from coarse to fine: (1) the 'material mesh' or 'phase mesh' (see Fig. 1a), (2) the 'elastic identification mesh' (see Fig. 1b), (3) the 'plastic identification mesh' (see Fig. 1c) and (4) the 'DIC mesh' (see Fig. 1d).

The 'material mesh' example in Fig. 1a shows that the grains were grouped in four phases corresponding to four material areas. In case of a heterogeneous material involving $n_{k}$ material domains, the CEG functional reads:

$$
\begin{gathered}
F_{C E G M}\left(\overrightarrow{u_{n}^{c}}, \underline{\underline{B_{n}^{s}}}\right)=\sum_{k=1}^{n_{k}} \sum_{n=1}^{N} \int_{\Omega_{k}}\left[\frac{\varepsilon}{\left.\left(\overrightarrow{u_{n}^{c}}\right)-\underline{\varepsilon}\left(\overrightarrow{u_{n}^{m}}\right)\right]: B_{\underline{k, n}}^{s}}\right. \\
:\left[\underline{\varepsilon}\left(\overrightarrow{u_{n}^{c}}\right)-\varepsilon\left(\overrightarrow{u_{n}^{m}}\right)\right] d \Omega=\sum_{k=1}^{n_{k}} F_{C E G M}^{k}\left(\overrightarrow{u_{n}^{c}}, \underline{\underline{k, n}}\right)
\end{gathered}
$$

where $B_{k, n}^{s}$ represents the secant tensor in the $k^{\text {th }}$ material domain at time step $n$. Note that this quantity also depends on the position in the case of plasticity.

\section{Elastic Properties Identification}

At a given load step $N$, the elastic properties identification consists first in estimating the stress field $\sigma_{n}^{c}$ at any $1 \leq n \leq N$ starting from a given mechanical property field and second in updating the property field in order to reduce the CEG cost function $F_{C E G M}$. These two operations are iteratively applied until convergence.

\section{Calculation of the Stress Field $\underline{\sigma_{n}^{c}}$}

At any load step $1 \leq n \leq N$, the measured displacement field $\overrightarrow{u_{n}^{m}}$, the location of the stress-free and loaded boundaries and the overall loadings are assumed to be known. The CEG functional is minimized with respect to the displacement field $\overrightarrow{u_{n}^{c}}$. We underline that at each loading step $n$, the FE solution of a heterogeneous elastic problem is used to compute the displacement field in both elasticity and plasticity. The algorithm is initialized with a set of non-vanishing arbitrary material parameters leading to an initial elastic stiffness tensor $\underline{\underline{B_{0}}}$.

The form of the constitutive law was here given by the equation (17) with $K_{n}=0$. Before starting the minimization, the displacement fields $\overrightarrow{u_{n}^{m}}$ were projected (in the least-squares sense) for each loading step on the elastic mesh. Since the 'elastic' mesh was, by construction, coarser than the DIC mesh, this projection consisted in filtering the displacements obtained by correlation, with the characteristic size of the filter being linked to the scale ratio between the two meshes. The cost functional was then minimized with respect to its first argument under the global and local equilibrium assumptions:

$\overrightarrow{u_{n}^{c}}=\operatorname{argmin} F_{C E G M}\left(, \underline{\underline{B_{n}^{s}}}\right)$

This first step in the minimization process allows the selection of one of many possible displacement fields, $\overrightarrow{u_{n}^{c}}$ and the corresponding standard admissible stress field, $\sigma_{n}^{c}$.

\section{Determination of the material properties}

After computing the set of displacement fields $\overrightarrow{u_{n}^{c}}$, the stress $\frac{\sigma_{n}^{c}}{n}$ is computed from the behavior law. In elasticity, the determination of material properties is explicit and corresponds to the stationarity of the CEG functional $F_{C E G M}$ with respect to the material parameters.

For plane displacement measurements and a given material domain $(k)$, the stationarity of $F_{C E G M}^{k}$ with respect to its second argument leads to three equations allowing the determination of the elastic parameters $E_{k}, v_{k}$ and $G_{k}$. Equation (5) shows that the elasticity tensor can be written as a function of parameters $b_{1}, b_{2}$ and $b_{3}$. As proposed in [24,25], the material minimization is performed analytically using the $b_{i}$ parameters, and the elastic constants are determined by expressing $E_{k}$, $v_{k}$ and $G_{k}$ with respect to the $b_{i}$.

\section{Convergence criterion}

For a given load step $N$, the procedure is stopped when the following convergence criterion on the elasticity tensor is reached on every material domain $k$ ( $i$ and $i+1$ denote two successive iterations, $\epsilon_{e}$ is about 0.001 and $\|A\|_{2}$ stands for the L2-norm of any tensor $A$ ):

$$
\left\|\left(B_{k \underline{\underline{N}}}^{e}\right)_{i+1}-\left(B_{k \underline{\underline{N}}}^{e}\right)_{i}\right\|_{2}<\epsilon_{e}\left\|\left(B_{k \underline{\underline{N}}}^{e}\right)_{i+1}\right\|_{2}
$$

Moreover, during this elastic properties identification, the onset of plasticity is checked. 


\section{Detection of plasticity onset}

The detection of the last elastic load step, denoted $N_{e}$, consisted in estimating the variation in the elastic tensor over each material domain $k$ :

$\exists k,\left\|\underline{\underline{B_{k, N_{e}+1}^{e}}}-\underline{\underline{B_{N_{e}}^{e}}}\right\|_{2}>\epsilon_{p}\left\|\underline{\underline{B_{k, N_{e}+1}^{e}}}\right\|_{2}$

where $\epsilon_{p}$ was typically about 0.05 . The elastic parameters are updated to match those identified at this step, and we consider that the plasticity starts at step $N_{e}+1$. Once at least one material domain reached plasticity, the elastic identification procedure is stopped and the plastic identification starts. Consequently, any significant change in the identified secant tensor is related to the onset of plasticity. This strategy has two main advantages: (i) the plasticity detection is independent of any a priori for the yield stress, and (ii) it is defined by introducing a single parameter quantifying the relative change in the identified secant tensor. This change is independent from the chosen set of elastic parameters.

The minimization algorithm for the elastic identification is summarized in Fig. 2.

\section{Plastic Identification}

The plastic identification requires knowledge of the stress state through the (load-dependent and unknown) plastic parameter $K_{n}$ in order to estimate the secant tensor. Since this parameter $K_{n}$ depends on the local loading path, it is not constant over each material domain and has to be identified for each element of the stress mesh.

\section{Initial estimation of parameter $K_{n}$}

Assuming an overall radial and monotonic loading (see Figure 3), a secant elastic identification is performed at each plastic step, thus allowing a simultaneous access to the secant elastic stiffness tensor at various time steps $\left(n>N_{e}\right)$ for each material domain $(k)$ and a compatible stress field for each load step $n\left(\sigma_{n}^{c}\right)$. The corresponding parameters are denoted with the subscript "es" $\left(E_{k, n}^{e s}, \nu_{k, n}^{e s}\right.$ and $\left.G_{k, n}^{e s}\right)$ since they differ from the actual elastic constants $\left(E_{k}, \nu_{k}, G_{k}\right)$ identified at time step $N_{e}$.

At this stage, a plastic parameter $K_{k, n}$ can be estimated for each material domain $k$ and at each plastic step $n$. Assuming that the plastic properties are homogeneous at the initialization step over each material domain and equalizing for each domain $k$ and at each plastic step $n$ the identified secant elastic tensor to the secant elastoplastic tensor, the plastic parameter $K_{k, n}$ is found as the only unknown of a three equation system solved in a least squares sense:

$$
\left.\begin{array}{cc}
\frac{E_{k}\left(\nu_{k}+K_{k, n} E_{k}\right)}{3 K_{k, n} 2 E_{k} 2-2 K_{k, n} E_{k}\left(\nu_{k}-2\right)+1-\nu_{k} 2} & 0 \\
\frac{E_{k}\left(1+2 K_{k, n} E_{k}\right)}{3 K_{k, n} 2 E_{k} 2-2 K_{k, n} E_{k}\left(\nu_{k}-2\right)+1-\nu_{k} 2} & 0 \\
0 & \frac{2 G_{k}}{1+12 K_{k, n} G_{k}}
\end{array}\right]
$$

This leads via equation (14) to a first estimate of the secant elastoplastic tensor $\left(\underline{\underline{B_{k, n}^{s}}}\right)_{0}$ for all loading steps $n$ greater than $N_{e}$. Again, this first estimate of the secant elastoplastic tensor is assumed to be homogeneous over each material domain at each loading step.

Identification of Plastic Properties Once this secant elastoplastic tensor is initialized, the local plastic parameters and stress fields can be identified. The determination of the plastic parameters is performed in two consecutive minimizations: first with respect to the displacement field $\overrightarrow{u_{n}^{c}}$ associated with a statically admissible stress field $\underline{\sigma_{n}^{c}}$ and second with respect to the secant tensor $\underline{\underline{B_{n}^{s}}}$ to identify the material parameters. The first minimization is done in the same manner as during the elastic identification, while changing only the stress mesh (see Figure 1c). Once the displacement fields are calculated for all plastic loadings, the plastic parameters are determined via the second 'material' minimization. The second minimization step consists in minimizing $F_{C E G M}(1.20)$ with respect to parameters $a_{K}$ and $b_{K}$. Unlike the elastic identification, the obtained system of two equations with two unknowns is not linear. This system is numerically solved using a gradient-based algorithm. The existence and unicity of the solution is not ensured. If no solution is found or if the identified parameters are not physically acceptable (i.e. negative), the material domain is assumed to remain elastic. 
Fig. 2 Elastic identification algorithm for a given load step $\mathrm{N}$

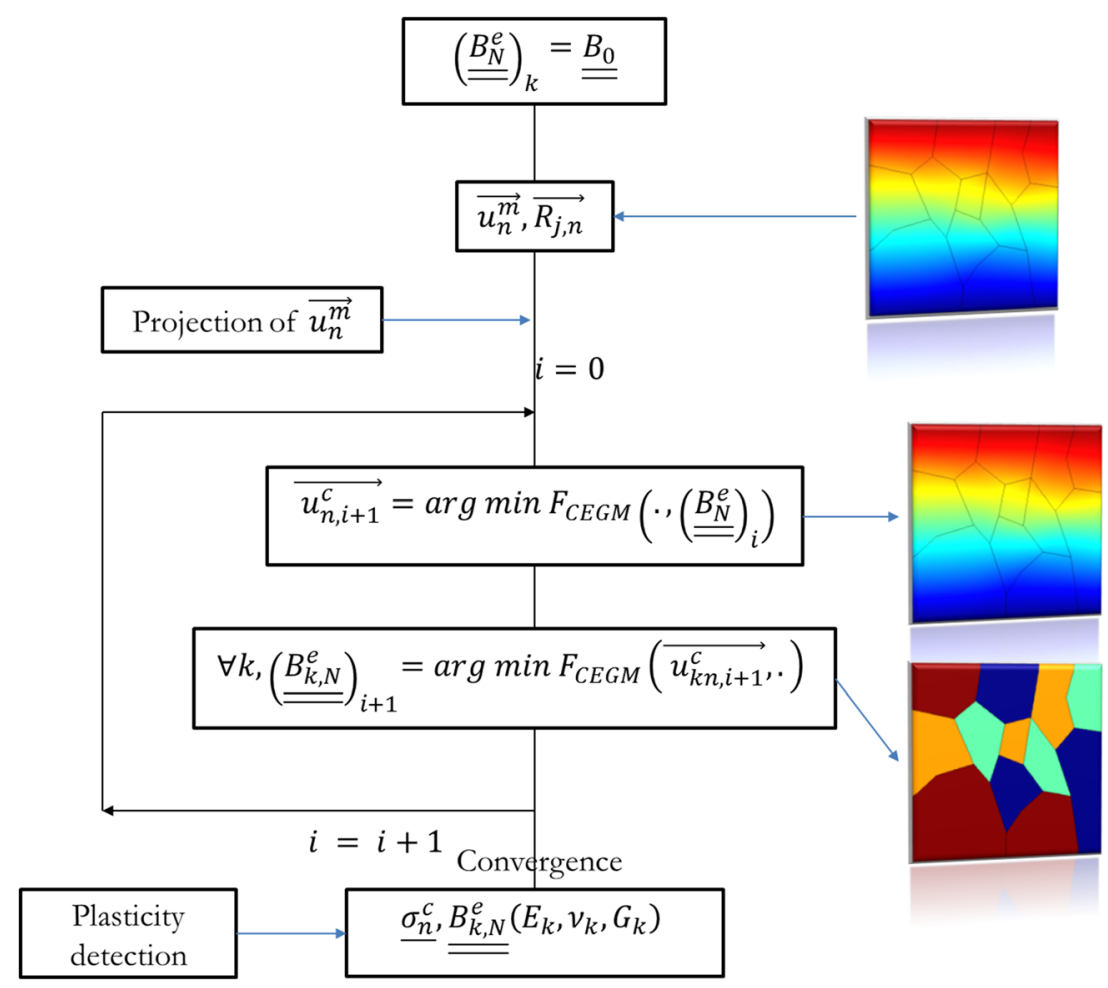

From the parameters $a_{K}$ and $b_{K}$ at a given load step, the local values of parameters $K$ and $\alpha$ are computed using the expressions (1.17), (1.18) and (1.19) on each plastic mesh element. In the case of a linear kinematic hardening model, it can be shown that the following relation is affine with a slope equal to $h$ (hardening modulus) and an ordinate at the origin equal to $\sigma_{0}$ (yield stress):

$\sqrt{\frac{3}{2}} \alpha_{n}^{i}=f\left(2 \sqrt{\frac{3}{2}} K_{n}^{i} \alpha_{n}^{i}\right)$

At any plastic time step $n>N_{e}, \sigma_{0}$ and $h$ are determined for each domain by performing a linear regression on the set of

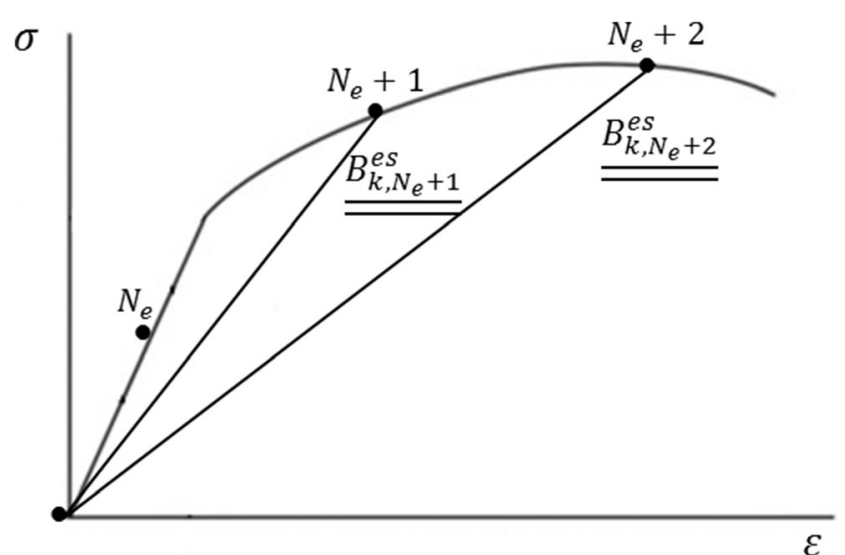

Fig. 3 Secant elastic identification for a given material domain $\mathrm{k}$ pairs $\left(2 \sqrt{\frac{3}{2}} K_{n}^{i} \alpha_{n}^{i}, \sqrt{\frac{3}{2}} \alpha_{n}^{i}\right)$ associated with all elements $i$ of the considered material domain. The quantity $2 \sqrt{\frac{3}{2}} K_{n}^{i} \alpha_{n}^{i}$ is the local equivalent plastic strain while $\sqrt{\frac{3}{2}} \alpha_{n}^{i}$ is the effective von Mises stress.

The graph in Figure 4 concerns a given material domain and each cross represents a calculated value, after convergence, for a given element of the plastic mesh at a given time step. The elements that weakly deform (using a criterion similar to that given in Equation (23)) are plotted as red circles and are not included in the linear regression. Here again, we introduce a threshold defined by reference to the maximum plastic deformation below which it is considered that the plastic deformation is too small to achieve a reliable identification. The theoretical answer (straight line) is superimposed in Figure 4.

This procedure is done iteratively until convergence using again a relative norm of the difference between the secant tensors identified at two consecutive iterations. At convergence, we obtain the stress tensor $\sigma_{n}^{c}$, the hardening modulus $h$, the yield stress $\sigma_{0}$, the secant elastoplastic tensor $\underline{B_{n}^{s}}$ and the fields $K_{n}$ and $\alpha_{n}$ for all plastic steps $n>N_{e}$. The iterative procedure is presented in Figure 5.

Multilinear Behavior The previous approach can be straightforwardly extended to the case of multilinear kinematic hardening since the expressions of the secant elastoplastic tensor (1.16 1.17) remain valid even if the behavior is not associated to a 
Fig. 4 Example of a curve $\alpha_{\mathrm{n}}^{\mathrm{i}}=$ $\mathrm{f}\left(\mathrm{K}_{\mathrm{n}}^{\mathrm{i}} * \alpha_{\mathrm{n}}^{\mathrm{i}}\right)$ obtained at two time steps in a heterogeneous test

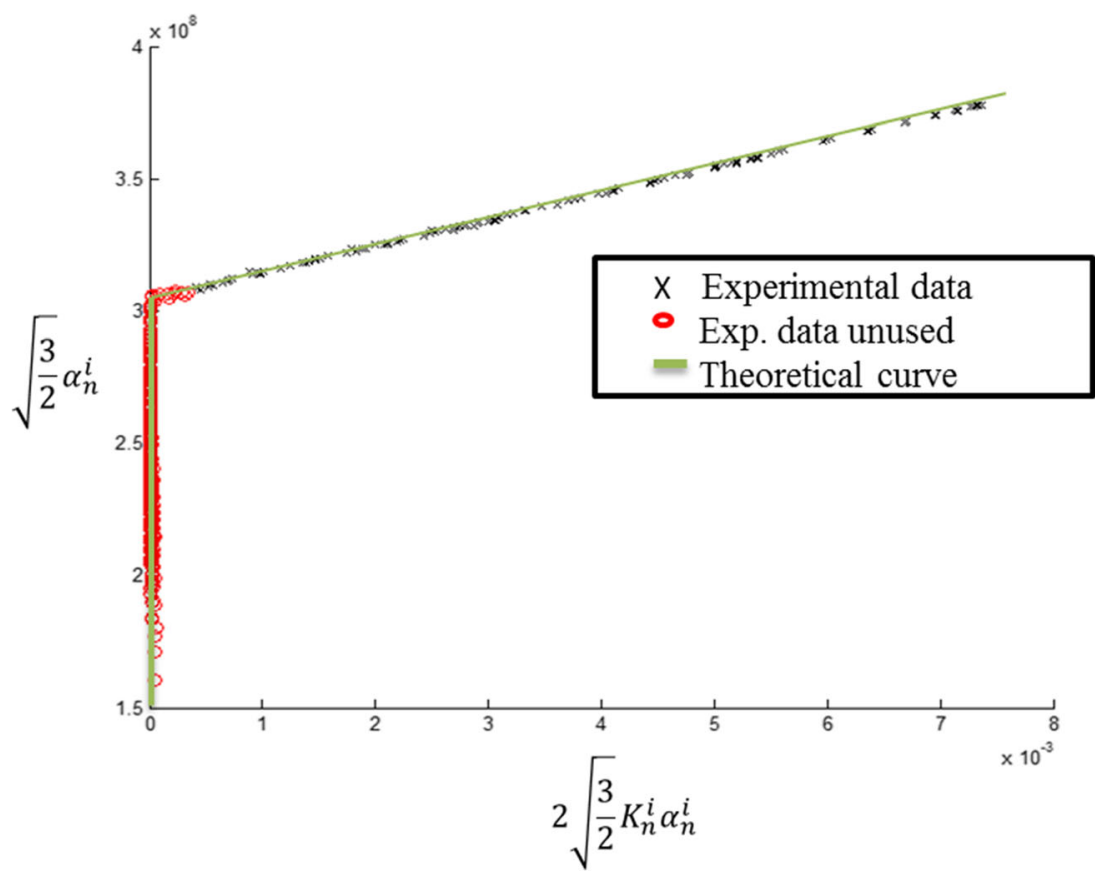

linear kinematic hardening, the curve $\left(\sqrt{\frac{3}{2}} \alpha_{n}^{i}, 2 \sqrt{\frac{3}{2}} K_{n}^{i} \alpha_{n}^{i}\right)$ corresponding then to the hardening law of the material.

At that point, we define the classes on which the multilinear hardening has to be determined. These classes correspond to the limits of the deformation intervals in which the hardening curve is discretized.

The Figure 6a shows the couples $\left(\sqrt{\frac{3}{2}} \alpha_{n}^{i}, 2 \sqrt{\frac{3}{2}} K_{n}^{i} \alpha_{n}^{i}\right)$ obtained after the initialization procedure of parameter
Fig. 5 Plastic identification algorithm

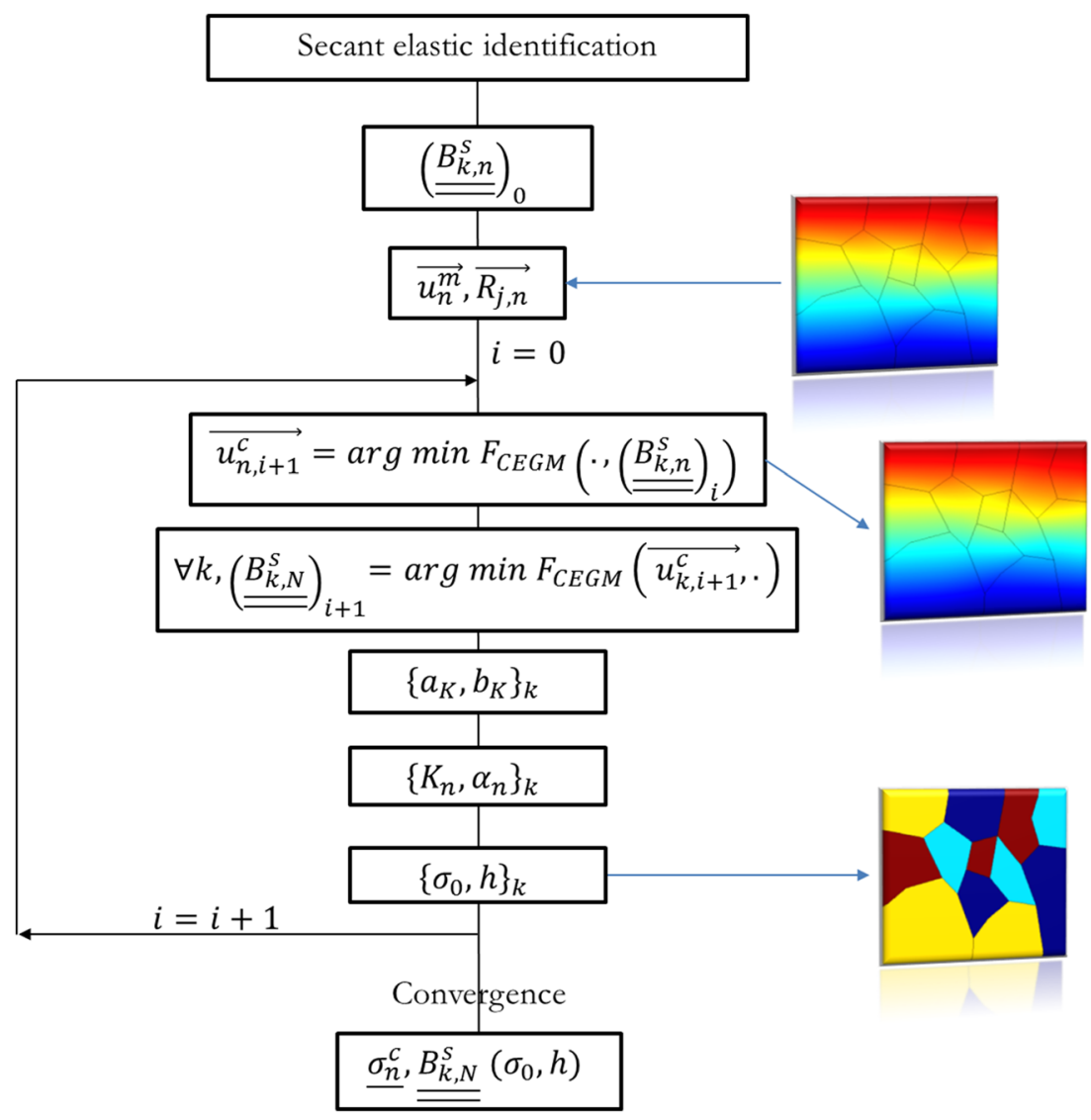


Fig. 6 a Identification data and hardening classes at the first iteration. $\mathrm{b}$ Example of a curve $\alpha=\mathrm{f}(\mathrm{K} * \alpha)$ obtained for a heterogeneous test on a material having bi-linear hardening (numerical test)
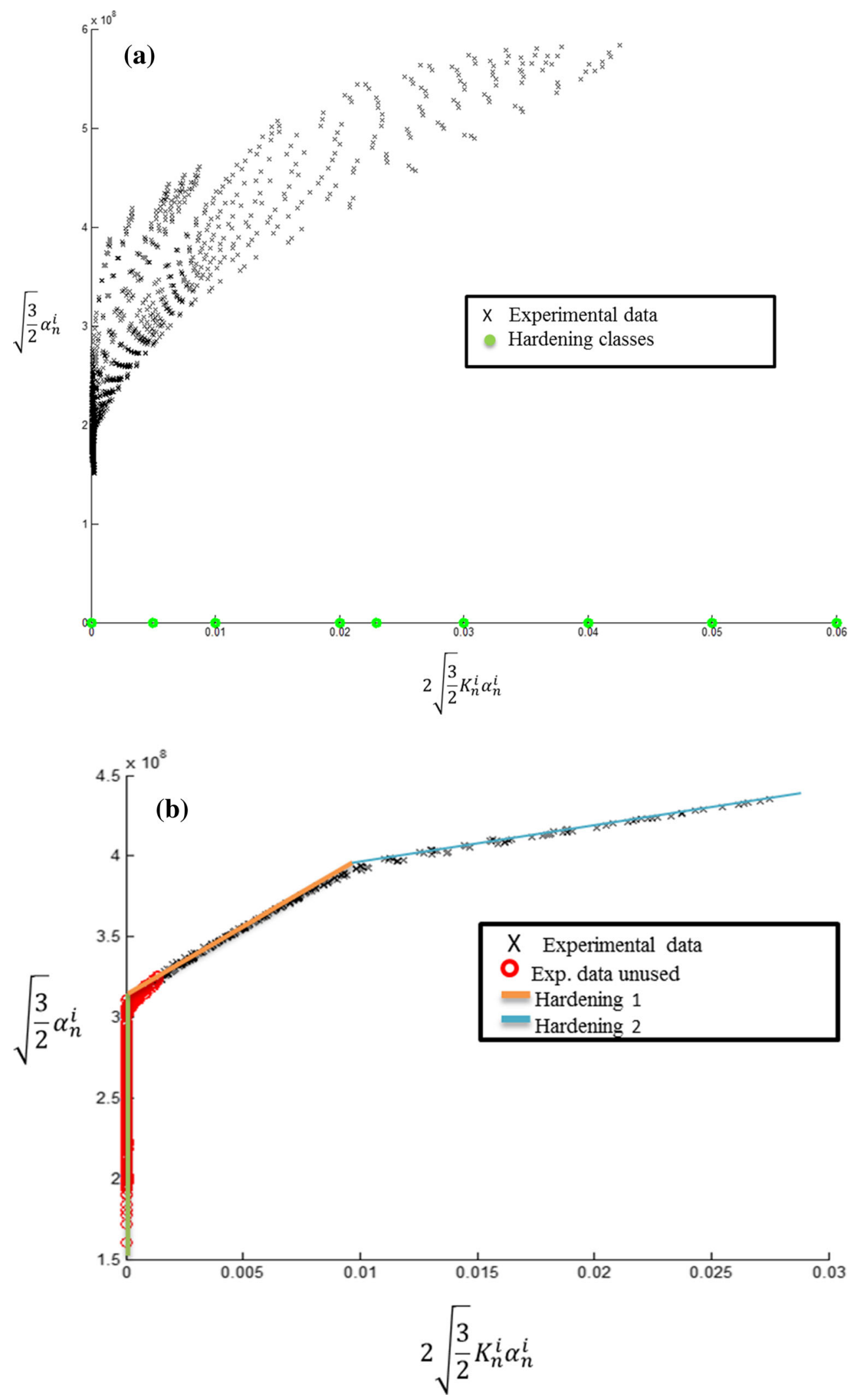

$K_{n}$. These values are obtained using FE-generated data associated with a homogeneous tensile test with bilinear hardening (yield stress $\sigma_{0}=300 \mathrm{MPa}, h_{1}=10 \mathrm{GPa}, \sigma_{1}=$
$400 \mathrm{MPa}, h_{2}=2 \mathrm{GPa}$ ) on a dog-bone specimen In this figure, the green circles represent the hardening classes used for the initialization of the multilinear 
identification. Note that the classes could be irregular. It is important to mention here that in this example the real transition between the two hardening regimes was compatible with the hardening initialization classes. With this choice, it was possible to capture the real material constants.

For a given iteration and for the $m^{\text {th }}$ class, we isolate the points in the plane $\left(\sqrt{\frac{3}{2}} \alpha_{n}^{i}, 2 \sqrt{\frac{3}{2}} K_{n}^{i} \alpha_{n}^{i}\right)$ belonging to that class and we identify the hardening characteristics $\left(k_{m}\right.$ and $\left.\sigma_{m}\right)$ in class $m$ by linear regression.

If the identified parameters of two constitutive classes ( $m$ and $m+1)$ are close enough, i.e.:

$\frac{\left|h_{m+1}-h_{m}\right|}{h_{m+1}}<\delta_{\text {hardening }}$

we considered that both classes corresponded to a single hardening and they are merged, otherwise they are considered as two different hardenings. The parameter $\delta_{\text {hardening }}$ is userdefined (typically 0.05 ) and quantifies the minimal hardening variation between two consecutive classes. The criterion used to quantify the hardening evolution can be modified to also take into account changes in the ordinate at the origin $\sigma_{m}$ between two consecutive classes.

Figure $6 \mathrm{~b}$ shows the couples $\left(\sqrt{\frac{3}{2}} \alpha_{n}^{i}, 2 \sqrt{\frac{3}{2}} K_{n}^{i} \alpha_{n}^{i}\right)$ obtained after convergence of the algorithm. This figure shows that the proposed method allows correct identification of the two hardening segments. This procedure enabled automatic determination of hardening segments in the material response.

In the next section, we present the validation of the procedure for identifying heterogeneous property fields using data from finite element simulations on different types of structures. These numerical data are intentionally corrupted using white Gaussian noise to check the robustness of procedure with respect to perturbations.

\section{Inverse Problem Resolution: Numerical Data}

In this section, the numerical simulations are conducted with COMSOL Multiphysics software to obtain manageable inputs for the identification procedure. The local in-plane components of the displacement field are extracted at the nodes of the finite element discretization and the overall loadings are extracted at the outer boundaries. The identification method is tested on numerical examples concerning both homogeneous or heterogeneous materials subjected to uniaxial tensile test. Moreover, the robustness of the method is illustrated by studying its sensitivity on noisy data.

\section{Finite Element Models}

The identifications are carried out on two numerical tests.

(i) The first test (specimen 1) concerned a standard tensile test performed at constant velocity on an isotropic elastoplastic material (Young modulus $\mathrm{E}=210 \mathrm{GPa}$, Poisson ratio $\mathrm{v}=0.3$, yield stress $\sigma_{-} 0=300 \mathrm{MPa}$ and hardening modulus $\mathrm{h}=1 \mathrm{GPa}$ ). The material parameters are identified using data associated with four load steps (one in the elastic domain, and three in the plastic one). Although the material was homogeneous, the identification is conducted in four material domains to illustrate the ability of the method to deal with low contrast materials (Figure 7).

(ii) The second test (specimen 2) corresponds to a polycrystalline metallic material consisting of four phases (" $\mathrm{R}$ " red, "O" orange, "Y" yellow and "G" green) randomly distributed in a tessellation and associated with four different sets of material parameters (Figure 8).

As previously mentioned, four different meshes are used for the identification method (see Figure 9 for the first test).

Different meshes are used for the direct computation of the reference displacement fields and for the inverse identification. However, the modeling error is cancelled by using the same mechanical models in the direct computation and in the identification procedure. The choice of DIC mesh and stress mesh (definition of refinement ratios in particular) is crucial in the identification process. The DIC mesh has to be chosen to limit DIC measurement errors (model error, interpolation error, etc.) [35], the stress mesh (elastic and plastic) has to be fine enough to catch the stress gradients and the material mesh has to be compatible with the heterogeneity of the material.

$$
B=\left\{\begin{array}{c}
E=210 \mathrm{GPa} \\
v=0.3 \\
\sigma_{0}=300 \mathrm{MPa} \\
h=1 \mathrm{GPa}
\end{array}\right.
$$

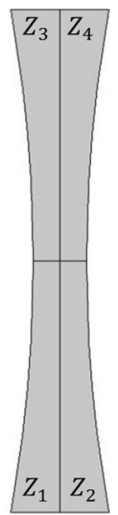

Fig. 7 Geometry and properties of the first specimen $(300 \mathrm{~mm}$ radius, $100 \mathrm{~mm}$ height, $10 \mathrm{~mm}$ width at the center and $1 \mathrm{~mm}$ thickness) 

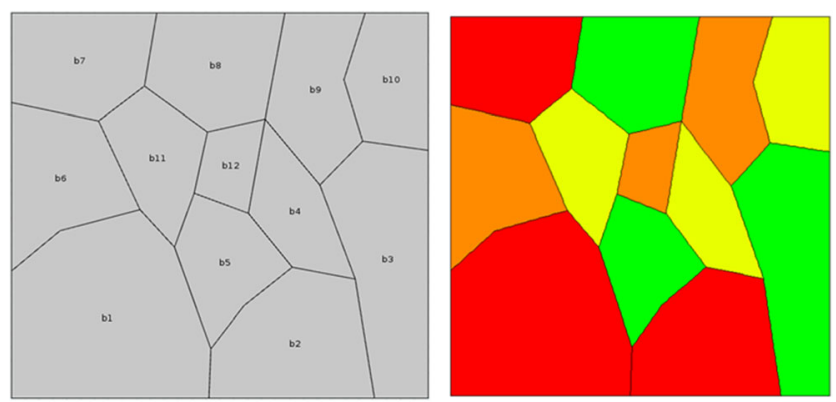

$$
B^{R}=\left\{\begin{array}{c}
E^{R}=210 \mathrm{GPa} \\
v^{R}=0.3 \\
\sigma_{0}^{R}=300 \mathrm{MPa} \\
h^{R}=2 \mathrm{GPa}
\end{array}\right.
$$$$
B^{O}=\left\{\begin{array}{c}
E^{O}=180 \mathrm{GPa} \\
v^{O}=0.25 \\
\sigma_{0}^{O}=300 \mathrm{MPa} \\
h^{o}=2.5 \mathrm{GPa}
\end{array}\right.
$$

$$
B^{Y}=\left\{\begin{array}{c}
E^{Y}=150 \mathrm{GPa} \\
v^{Y}=0.2 \\
\sigma_{0}^{Y}=300 \mathrm{MPa} \\
h^{Y}=1.5 \mathrm{GPa}
\end{array}\right.
$$$$
B^{G}=\left\{\begin{array}{c}
E^{G}=100 \mathrm{GPa} \\
v^{G}=0.15 \\
\sigma_{0}^{G}=300 \mathrm{MPa} \\
h^{G}=1 \mathrm{GPa}
\end{array}\right.
$$

Fig. 8 Geometry and properties of the polycrystalline specimen consisting of four phases (R, O, Y, and $\mathrm{G}$ ) and 12 grains (b1 to b12)

For the direct computation, the constant vertical displacement is prescribed on the lower and the upper boundaries of the specimen, in-plane rigid body motion are avoided prescribing the horizontal displacement of one node of the structure, and the other boundaries are stress-free. These boundary conditions are applied to both geometries tested in this section. Direct calculations are performed under small perturbation and plane stress

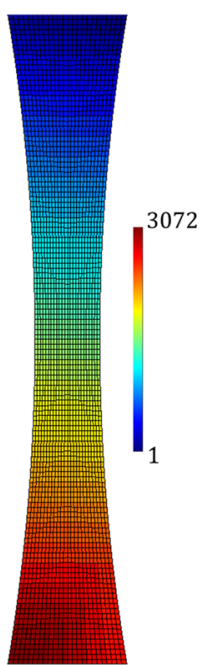

(a)

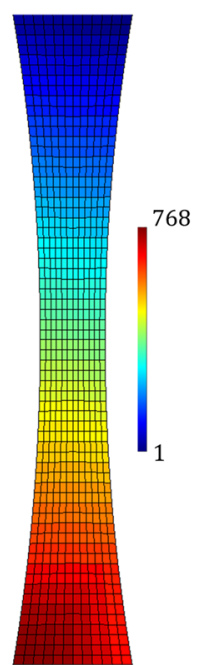

(b)

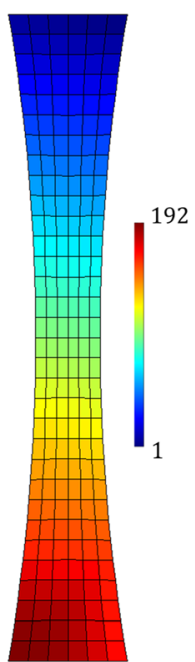

(c)

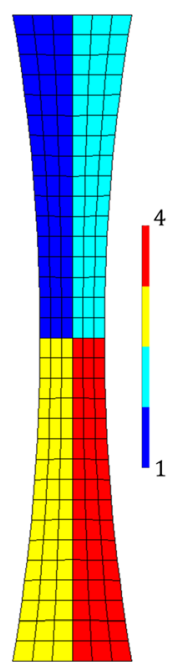

(d)
Fig. 9 First specimen: 'DIC' mesh with 3072 quadrangular elements used to obtain the measured displacement fields denoted $\overrightarrow{u_{n}^{m}}$ at each loading step (a), 'plastic' mesh with 768 elements used to describe the stress field in the plastic identification step. (b), 'elastic' mesh with 192 elements used to describe the stress fields in the elastic identification (c) and four material domains (d) assumptions. The investigated mechanical properties correspond to isotropic elastoplastic behavior with linear or multilinear kinematic hardening.

\section{Homogeneous Structure with Heterogeneous Fields: First Specimen}

The shape of specimen 1 provides heterogeneous mechanical fields for a homogeneous material and thus allows a validation of the method in a slightly heterogeneous situation. This specimen is also used to analyze the sensitivity of the identification method on the identification mesh, on the initialization parameters and on the measurement noise.

\section{Identification on identical meshes}

First we use identification meshes (displacement and stress) similar to the one used for direct calculation. The geometry is divided into four material domains $\left(Z_{1}, Z_{2}, Z_{3}, Z_{4}\right)$ (Figure 10a) wherein the material parameters are assumed to be constant while the direct computation is performed on a homogeneous material. The identification is performed at one load step in the elastic response and at three steps in the plastic response (see time-strength curve in Figure 10b). The initial values in the elastic identification algorithm are set to about the half of the values imposed in the direct calculation $\left(E_{0}=\right.$ $100 \mathrm{GPa}$ et $v_{0}=0.15$ ). No initial values have to be chosen for plastic parameters since they are determined during the secant elastic identification.

Table 1 summarizes the results of the elastoplastic identification for the four material domains $\left(Z_{1}\right.$ to $\left.Z_{4}\right)$. The identified values are very close to the reference values. The maximum relative error is about $3 \%$ for the hardening modulus $k$ (otherwise for a single material domain it is about $1 \%$ ). The elastic constants and the elastic limit are identified with very good accuracy (less than $0.5 \%$ relative error).

One loading step is sufficient to identify elastic properties but considering more loading steps can improve the accuracy of the linear identification in the case of noisy experimental data. For the plastic identification, three loading steps are here overabundant since a low heterogeneity is expected in the mechanical fields and a single step could have been possible. The identified parameters corresponded to a homogeneous material: the four material domains (zones 1-4) are identified with very similar coefficients $(3 \%$ maximum relative error, Table 1). The identification procedure converges in 6-8 iterations (elastic and plastic) and the calculation time is about 8$10 \mathrm{~min}$ for complete identification (calculation performed on a HP Z820 Workstation).

In the sequel, we systematically represent the mean stress per element. Figure 11a and $\mathrm{b}$ show the accuracy between the axial stresses obtained by direct calculation $\left(\underline{\sigma_{y y}^{m}}\right)$ and by 
Fig. 10 Material meshes (a) and load steps used for identification (b)

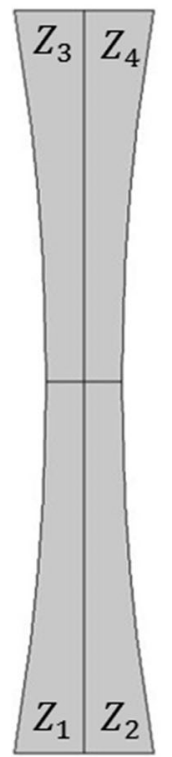

(a)

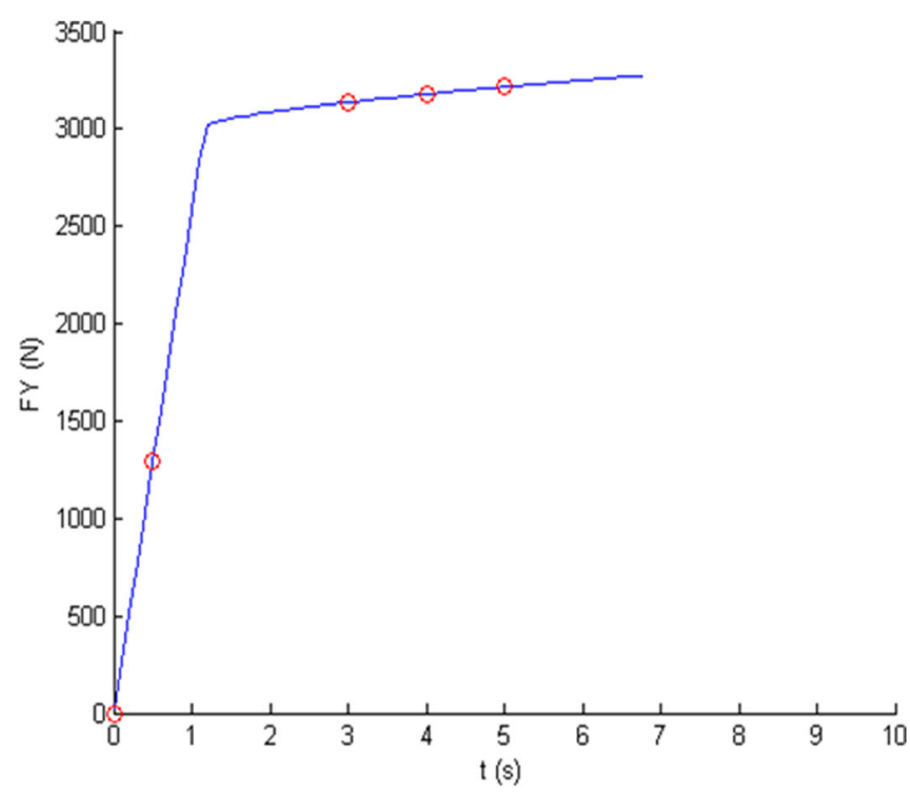

(b) identification $\left(\underline{\sigma_{y y}^{c}}\right)$, the relative error being less than $3 \%$. Figure 11c shows the von Mises stress of the absolute error. Note that the highest error levels are located in the corners of the specimen where the stress gradients are the strongest. These errors can be explained by the fact that the meshes used for the 'elastic' (192 elements) and 'plastic' (768 elements) identifications are coarser than those used for the simulation (3072 elements).

\section{Sensitivity to the initial set of the elastic identification}

The method only required the choice of a set of initial material parameters. The sensitivity of the procedure to these initial values is checked on three initial sets. The convergence speed is also highlighted. The material constants are identified on a single material domain. The results are presented in Table 2 .

Table 1 Parameters identified by the inverse method

\begin{tabular}{lllll}
\hline Parameters & $E(G P a)$ & $v$ & $k(G P a)$ & $\sigma_{0}(\mathrm{MPa})$ \\
\hline Reference value & 210.00 & 0.30 & 1.00 & 300.00 \\
$\mathrm{Z}_{1}$ & 209.80 & 0.30 & 1.03 & 298.49 \\
Relative error (\%) & 0.10 & 0.00 & 3.00 & 0.50 \\
$\mathrm{Z}_{2}$ & 210.05 & 0.30 & 0.99 & 299.51 \\
Relative error (\%) & 0.02 & 0.00 & 1.00 & 0.16 \\
$\mathrm{Z}_{3}$ & 210.12 & 0,30 & 1.00 & 299.38 \\
Relative error (\%) & 0.06 & 0.00 & 0.00 & 0.21 \\
$\mathrm{Z}_{4}$ & 209.86 & 0.30 & 0.99 & 299.60 \\
Relative error $(\%)$ & 0.07 & 0.00 & 1.00 & 0.13 \\
\hline
\end{tabular}

The three sets of initial values correspond to: (i) a very soft material, (ii) a material with consistent elastic properties (i.e. in the correct order of magnitude compared to the reference values), and (iii) a non-physical material (Poisson ratio greater than 0.5). In general, deviations in the identified values are very small (less than $0.1 \%$ ), except for the hardening modulus (about 3\%). They correspond to less than $1 \mathrm{MPa}$ for the elastic modulus and for the yield stress. They reach about tens of MPa in the hardening modulus. This table shows that the identified parameters are in good agreement with the reference values whatever the initial set and that the identification procedure is stable with respect to this initial set. In addition, the computational time is not significantly affected by the initial set choice.

\section{Sensitivity to mesh size}

The dependence of the identification procedure according to the mesh size turns now to be investigated in specimen 1 . An identical mesh is chosen for the elastic, plastic and DIC identification. Three different mesh sizes (coarse, normal and fine) are investigated. The mesh used for the direct computation has 2944 bi-linear quadrangular elements, while the coarse, normal and fine meshes have respectively 358, 1432 and 1958 quadrangular elements. Table 3 summarizes the obtained results.

The main conclusion is that the use of a finer mesh improved the plastic identification results since stress gradients are more accurately estimated. When the identification stress mesh is similar to that used for the direct computation, the identification error tends to vanish. As expected, the computation time increases with size of the problems. 
Fig. 11 Transversal stress of the last loading step obtained by: FE calculation $\sigma_{\mathrm{yy}}^{\mathrm{m}}(\mathrm{a})$ and by identification $\sigma_{\text {yy }}^{\mathrm{c}}(\mathrm{b})$ and the von

Mises stress of the absolute error (c)

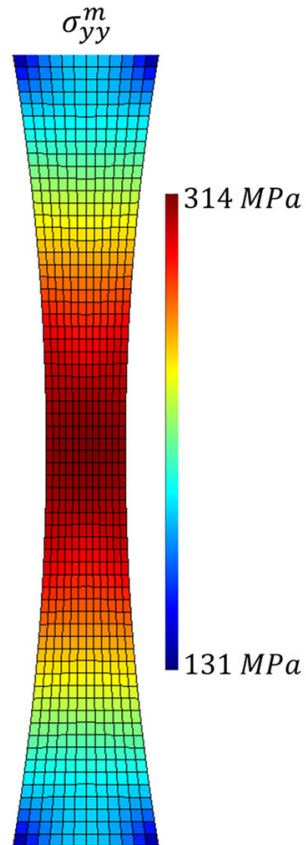

(a)

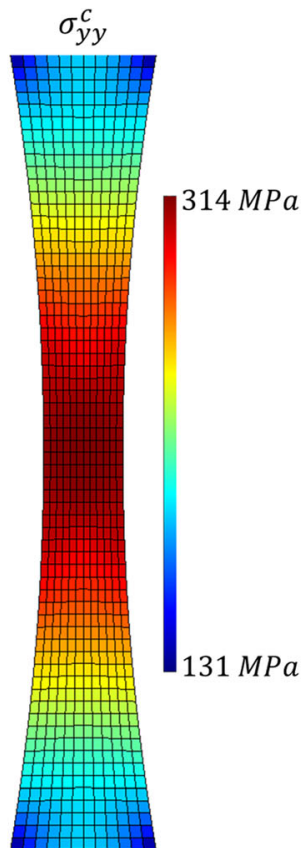

(b)

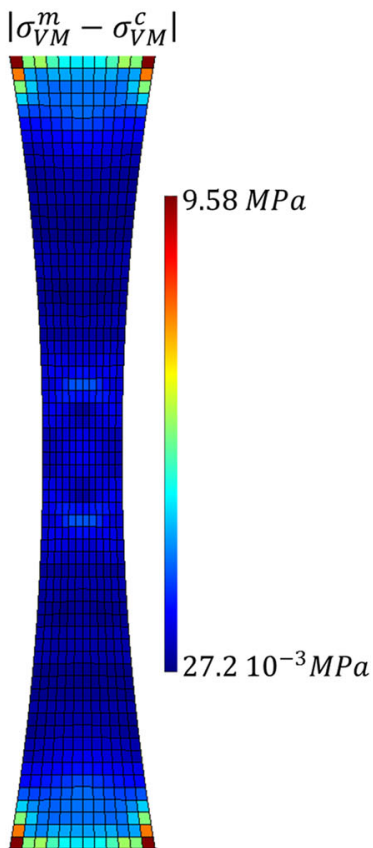

(c)

\section{Robustness to measurement noise}

The above results (sections 4.2.1 to 4.2.3) were obtained using the displacement fields directly extracted from the FE simulation. Since experimental measurements are never noise-free and inverse methods are known to be very sensitive to noise, we add here to FE-computed displacement fields some Gaussian white noise (standard deviation $\gamma$ ). Three different noise amplitudes are investigated: the "reference" noise level $\left(\sigma_{u}=\sigma_{u}^{r}=0.01\right.$ pixels $)$, the "low" noise level $\left(\sigma_{u}=2 \sigma_{u}^{r}\right)$ and the "high" noise level $\left(\sigma_{u}=4 \sigma_{u}^{r}\right)$. The conversion from metric dimension to pixels is performed based on the assumption that the specimen length corresponded to the size of the

Table 2 Sensitivity to the initial settings: specimen 1

\begin{tabular}{lllll}
\hline Parameters & $E(G P a)$ & $v$ & $k(G P a)$ & $\sigma_{0}(M P a)$ \\
\hline Reference value & 210.00 & 0.30 & 1.00 & 300.00 \\
Initial value 1 & $1.00 \mathrm{e}-09$ & $1.00 \mathrm{e}-09$ & $\ldots \ldots \ldots$ & $\ldots \ldots \ldots$ \\
Identified value & 209.74 & 0.30 & 1.03 & 300.10 \\
Relative error $(\%)$ & 0.12 & 0.00 & 3,00 & 0,03 \\
Iterations & 7 & 7 & 8 & 8 \\
Initial value 2 & 155.00 & 0.15 & $\ldots \ldots \ldots$ & $\ldots \ldots .$. \\
Identified value & 209.77 & 0.30 & 1.03 & 300.10 \\
Relative error $(\%)$ & 0.11 & 0.00 & 3.00 & 0.03 \\
Iterations & 7 & 7 & 6 & 6 \\
Initial value 3 & 420.00 & 0.60 & $\ldots \ldots \ldots$ & $\ldots \ldots \ldots$ \\
Identified value & 209.84 & 0.30 & 1.03 & 300.09 \\
Relative error $(\%)$ & 0.08 & 0.00 & 3.00 & 0.03 \\
Iterations & 8 & 8 & 6 & 6 \\
\hline
\end{tabular}

CCD sensor, i.e. here 4864 pixels. The "reference" noise level is consistent with typical errors obtained by DIC for weakly heterogeneous fields [26].

A statistical analysis is performed by comparing the identification results obtained using 50 different draws of displacement noise, and computing the average and standard deviation of the identification error on the material constants. Focusing on this noise error, we perform the analysis on a rectangular sample submitted to a uniform uniaxial stress field. The rectangle dimensions are chosen in accordance to the other specimen dimensions. The identification study is performed using one material domain. The finite element mesh is identical to the mesh used to describe the displacements obtained by correlation. These two grids consist in 448 quadrangular elements. The mesh includes 28 elements for elastic identification and 112 elements for plastic identification. Two cases are

Table 3 Sensitivity to the mesh density

\begin{tabular}{lllll}
\hline Parameters & $E(G P a)$ & $v$ & $k(G P a)$ & $\sigma_{0}(\mathrm{MPa})$ \\
\hline $\begin{array}{l}\text { Reference value } \\
\text { Coarse mesh }\end{array}$ & 210.00 & 0.30 & 1.00 & 300.00 \\
$\begin{array}{l}\text { Identified value } \\
\text { Relative error (\%) }\end{array}$ & 209.42 & 0.30 & 1.03 & 300.09 \\
Normal mesh & & 0.00 & 3.00 & 0.03 \\
Identified value & 209.94 & 0.30 & 0.99 & 302.79 \\
Relative error (\%) & 0.03 & 0.00 & 1.00 & 0.93 \\
$\begin{array}{l}\text { Fine mesh } \\
\text { Identified value }\end{array}$ & 209.94 & 0.30 & 1.00 & 299.63 \\
Relative error (\%) & 0.03 & 0.00 & 0.00 & 0.12 \\
\hline
\end{tabular}


Table 4 Sensitivity of the method to experimental noise: without filtering

\begin{tabular}{|c|c|c|c|c|c|}
\hline & $\begin{array}{l}\text { Parameters } \\
\text { Reference value }\end{array}$ & $\begin{array}{l}E(G P a) \\
210.00\end{array}$ & $\begin{array}{l}v \\
0.30\end{array}$ & $\begin{array}{l}k(G P a) \\
1.00\end{array}$ & $\begin{array}{l}\sigma_{0}(M P a) \\
300.00\end{array}$ \\
\hline \multirow{3}{*}{$\begin{array}{l}\text { Noise } \\
\text { Amplitude: } \mathbf{1} * \sigma_{\boldsymbol{u}}\end{array}$} & Mean identified value & 184.48 & 0.24 & 1.03 & 299.66 \\
\hline & Relative error (\%) & 12.15 & 20 & 3 & 0.11 \\
\hline & Standard deviation & $1.93 \mathrm{GPa}$ & 0.01 & $33.5 \mathrm{MPa}$ & $0.67 \mathrm{MPa}$ \\
\hline \multirow{3}{*}{$\begin{array}{l}\text { Noise } \\
\text { Amplitude: } \mathbf{2} * \sigma_{u}\end{array}$} & Identified value & 142.27 & 0.16 & 1.12 & 298.70 \\
\hline & Relative error $(\%)$ & 32.26 & 46.66 & 12 & 0.43 \\
\hline & Standard deviation & $3.10 \mathrm{GPa}$ & 0.02 & $100 \mathrm{MPa}$ & $1.81 \mathrm{MPa}$ \\
\hline \multirow{3}{*}{$\begin{array}{l}\text { Noise } \\
\text { Amplitude: } \mathbf{4} * \sigma_{u}\end{array}$} & Identified value & 87.23 & 0.09 & 1.47 & 294.84 \\
\hline & Relative error $(\%)$ & 58.46 & 70 & 47 & 1.72 \\
\hline & Standard deviation & $2.82 \mathrm{GPa}$ & 0.02 & $160 \mathrm{MPa}$ & $2.77 \mathrm{MPa}$ \\
\hline
\end{tabular}

investigated. The first one consists in direct use of the noisy displacements for identification while the second one used filtered displacements (projection in a least square sense of the noisy displacements on the stress elements).

Table 4 shows the results obtained without filtering. Naturally, the identification error (average and standard deviation) increases with the noise amplitude. The error on the elastic constants is significantly higher than for the plastic constants: for a fixed noise level, the signal-to-noise ratio is lower in the elastic case (in metallic materials, plastic deformations are typically about $10^{-2}$, while elastic deformation is about $10^{-3}$ ). As often observed, the Poisson ratio is more sensitive to noise. For the reference noise level, the identification error on the elastic constants is relatively high (greater than $10 \%$ ), while the error on the plastic constants is more acceptable (less than 3\%). This result can be explained by two reasons. First, the relative error on the elastic deformation is higher than the one associated with plastic deformation. Second, the plastic mesh is finer than the elastic mesh; consequently, one can expect the stress field to be better approximated using the (fine) plastic mesh than the (coarse) elastic mesh.
Table 5 shows the results obtained by keeping the same stress discretization and filtering the displacement data. It shows that the identification precision was improved (around $6 \%$ maximum relative error on the elastic constants). The filtering improved the identification while retaining the method's ability to describe heterogeneous stress fields.

\section{Identification of Heterogeneous Parameters and Stress Distribution: Coarse-Grained Polycrystalline}

In this section, we focus on identifying heterogeneous materials. The 12 polyhedral "grains" sample (see Figure 8) consists in four phases (each regrouping three grains) associated with different material parameters. The behavior of each phase is assumed to be isotropic with a J2-elastoplastic kinematic hardening behavior.

In this identification, we used a single load step for the elastic identification and ten load steps for the plastic identification (see Figure 12a). The mesh size used for the identification was different from the one used for the direct calculation.

Table 5 Sensitivity of the method to experimental noise: with filtering

\begin{tabular}{|c|c|c|c|c|c|}
\hline & $\begin{array}{l}\text { Parameters } \\
\text { Reference value }\end{array}$ & $\begin{array}{l}E(G P a) \\
210.00\end{array}$ & $\begin{array}{l}v \\
0.30\end{array}$ & $\begin{array}{l}k(G P a) \\
1.00\end{array}$ & $\begin{array}{l}\sigma_{0}(M P a) \\
300.00\end{array}$ \\
\hline \multirow[t]{3}{*}{ Noise Amplitude: $1 * \sigma_{u}$} & Identified value & 209.45 & 0.30 & 1.00 & 299.96 \\
\hline & Relative error (\%) & 0.26 & 0 & 0 & 0.01 \\
\hline & Standard deviation & $1.28 \mathrm{GPa}$ & 0.006 & $7.17 \mathrm{MPa}$ & $0.15 \mathrm{MPa}$ \\
\hline \multirow[t]{3}{*}{ Noise Amplitude: $2 * \sigma_{u}$} & Identified value & 207.97 & 0.29 & 1.00 & 299.92 \\
\hline & Relative error (\%) & 0.99 & 3.3 & 0 & 0.02 \\
\hline & Standard deviation & $2.58 \mathrm{GPa}$ & 0.01 & $13.3 \mathrm{MPa}$ & $0.26 \mathrm{MPa}$ \\
\hline \multirow[t]{3}{*}{ Noise Amplitude: $4 * \sigma_{u}$} & Identified value & 202.62 & 0.28 & 1.01 & 299.84 \\
\hline & Relative error (\%) & 3.51 & 6.6 & 1 & 0.05 \\
\hline & Standard deviation & $5.02 \mathrm{GPa}$ & 0.02 & $29.7 \mathrm{MPa}$ & $0.57 \mathrm{MPa}$ \\
\hline
\end{tabular}




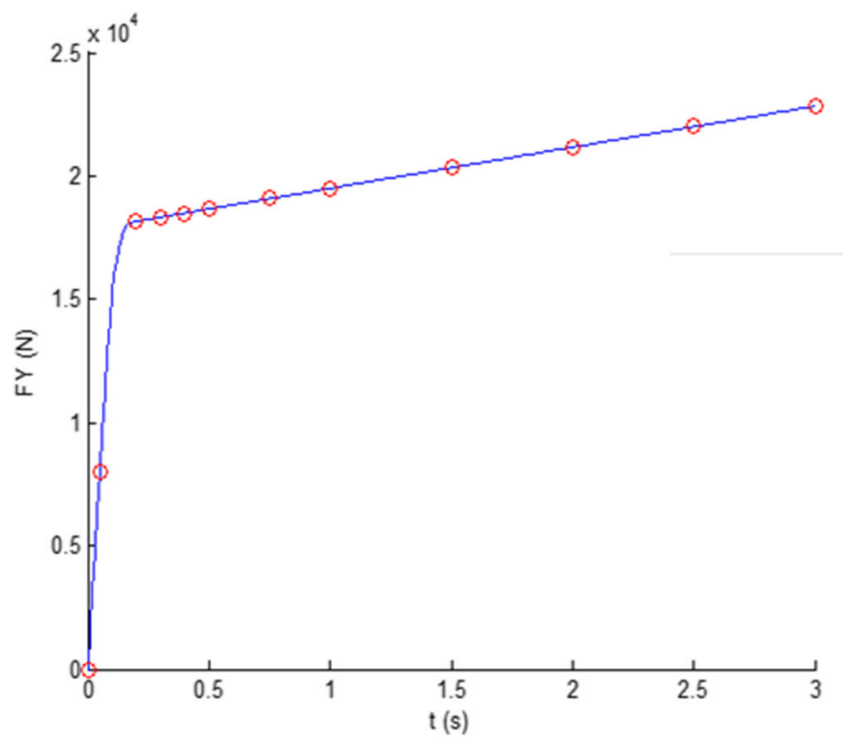

(a)

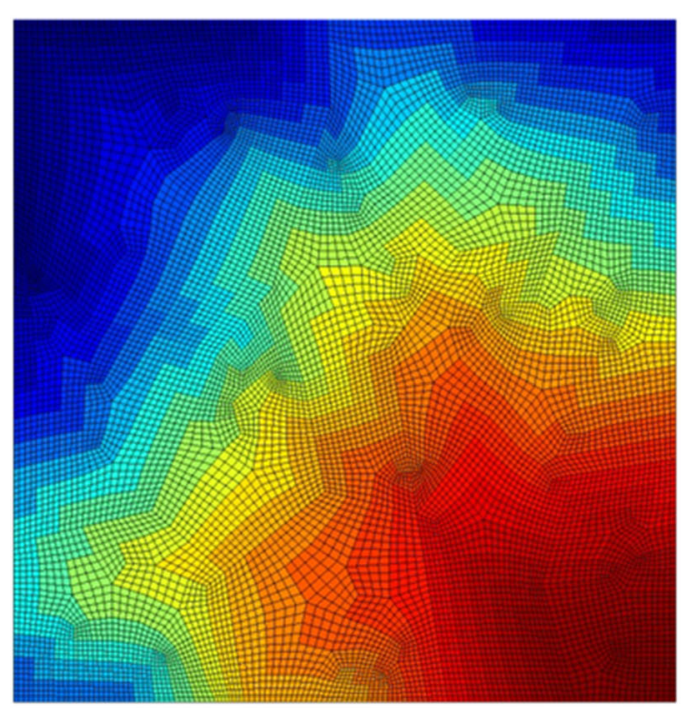

(b)

Fig. 12 Global loads and load steps used for identification (a) and mesh associated with the "measured" kinematic fields (b)

The mesh associated with the displacement fields consists in 13,072 elements (Figure 12b), while that used for the elastic identification has 817 elements and the mesh for the plastic identification consists in 3268 elements. The mesh of the direct computation had 15,049 bi-linear elements. We also assume that the phase distribution is known a priori (e.g. through EBSD analysis). We associate a material domain to each phase and identify the mechanical constants on each one. In Figure 13, we compare the reference Young's modulus and hardening modulus with respect to those identified.
Fig. 13 Comparison between identified and reference values of Young's Modulus and hardening modulus: Young's Modulus of reference (a) and the calculated (b), hardening modules of reference (c) and the calculated (d)

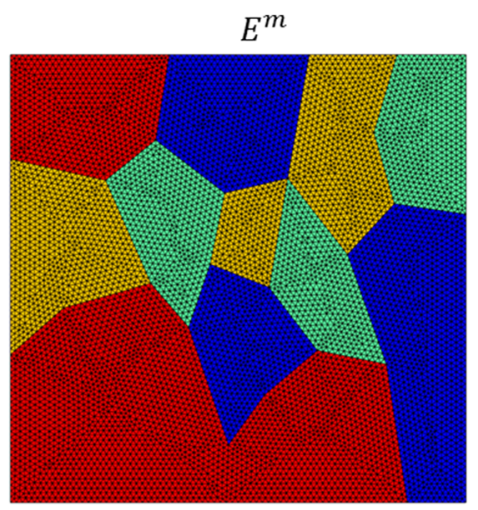

(a)

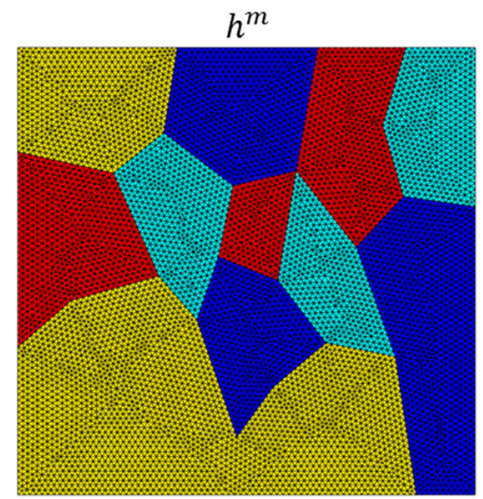

(c)

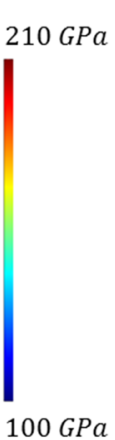

$100 \mathrm{GPa}$

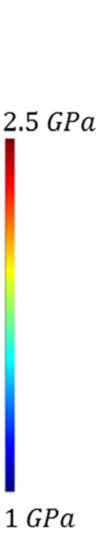

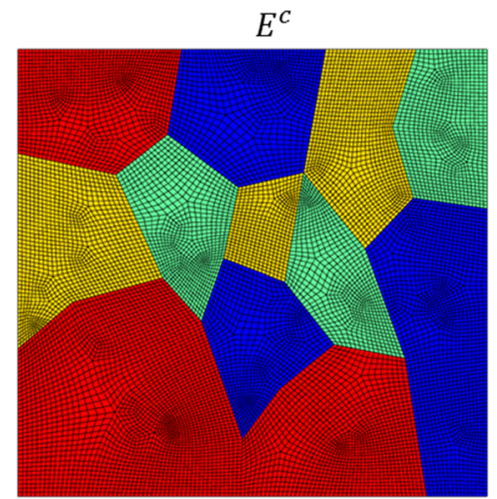

(b)

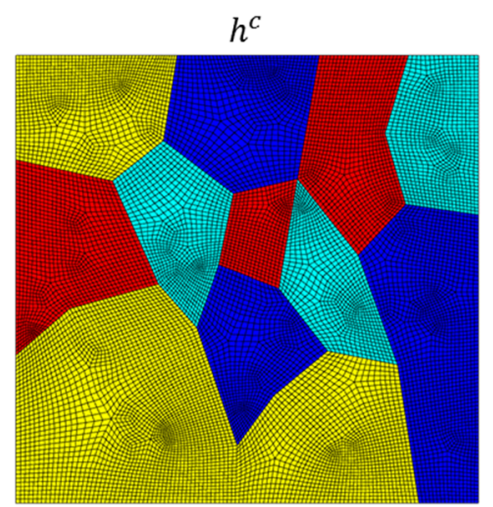

(d)

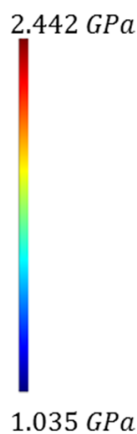

$208.22 \mathrm{GPa}$

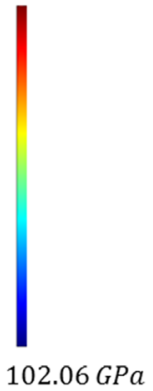

$102.06 \mathrm{GPa}$ 
Table 6 Parameters identified by the inverse method for the four polycrystal phases

\begin{tabular}{lcllc}
\hline Parameters & $E(G P a)$ & $v$ & $k(G P a)$ & $\sigma_{0}(M P a)$ \\
\hline Phase 1 & & & & \\
Reference value & 210.00 & 0.30 & 2.00 & 300.00 \\
$\quad$ Identified value & 208.218 & 0.298 & 1.963 & 299.831 \\
Relative error (\%) & 0.85 & 0.67 & 2.04 & 0.05 \\
Phase 2 & & & & \\
Reference value & 180.00 & 0.25 & 2.5 & 300.00 \\
Identified value & 178.058 & 0.249 & 2.442 & 299.585 \\
Relative error (\%) & 1.09 & 0.40 & 2.32 & 0.14 \\
Phase 3 & & & & \\
Reference value & 150.00 & 0.2 & 1.5 & 300.00 \\
Identified value & 151.226 & 0.200 & 1.539 & 300.670 \\
Relative error (\%) & 0.81 & 0.00 & 2.60 & 0.22 \\
$\quad$ Phase 4 & & & & \\
Reference value & 100.00 & 0.15 & 1.00 & 300.00 \\
Identified value & 102.062 & 0.152 & 1.035 & 301.013 \\
Relative error (\%) & 2.02 & 1.3 & 3.5 & 0.33 \\
\hline
\end{tabular}

In Figure 13d, a maximum relative error of about $2 \%$ is observed. The results of the identification for all parameters are shown in Table 6.

Table 6 and Figure 13d show that the largest identification errors were obtained with the hardening modulus. Table 6 indicates that, for each material domain, all the identified material parameters were either always overestimated or always underestimated.

Figure 14 compares the stress field identified at the last plastic step to the one computed by FE simulation at the same load step (reference field). The distribution and magnitude of the identified stress are similar to those of the stress from the FE simulation. Note, however, that the identified stress is slightly higher in some elements than the computed one. The stress gradients are properly transcribed except in areas of high stress gradients near the grain boundaries. The stress field identification is less accurate in the vicinity of triple points. The grain shape and the boundary orientations with respect to the loading direction have a significant influence on the stress distribution.

\section{Inverse Problem Resolution: Experimental Data}

This section presents the identification results obtained with the experimental data.

\section{Testing Conditions}

The sample (Figure 15) is machined on a $2.6 \mathrm{~mm}$ thick DP600 dual phase plate obtained by hot rolling. This material contains a hard phase of martensite dispersed in a ductile ferrite matrix. A typical grain size is about $10 \mu \mathrm{m}$ and the material can reasonably be assumed to be homogeneous in its initial state with elastoplastic behavior.

Figure 16 illustrates the prescribed mechanical loading. The tests consist in a series of velocity-controlled loading/
Fig. 14 Transversal stress of the last loading step obtained by: FE calculation $\sigma_{\text {yy }}^{\mathrm{m}}(\mathrm{a})$ and by identification $\underline{\sigma_{\mathrm{yy}}^{\mathrm{c}}(\mathrm{b})}$ and the von Mises stress of the absolute error (c)

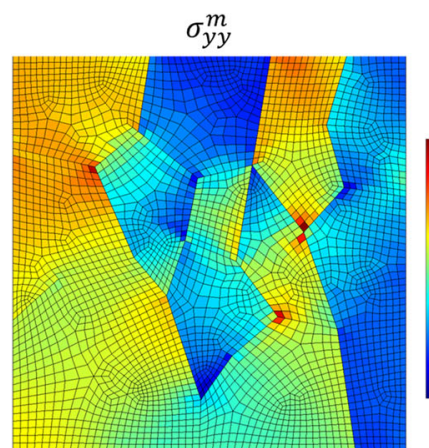

(a)

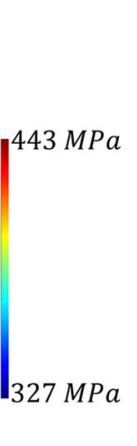

.

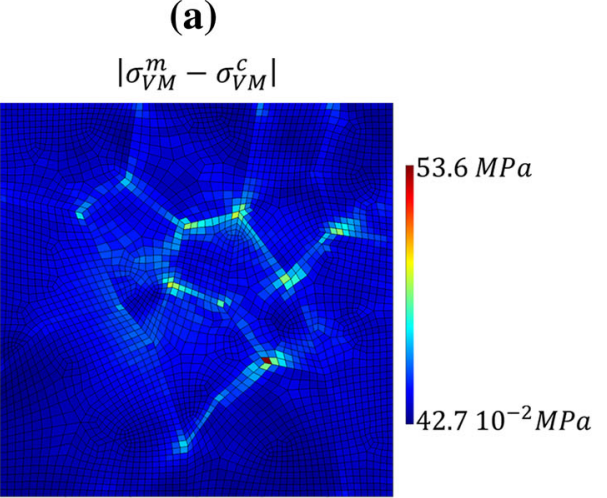

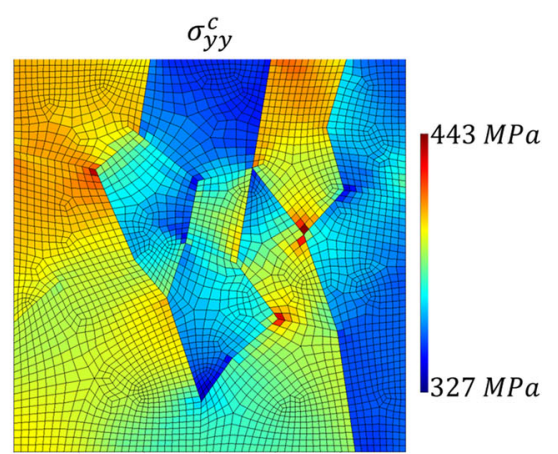

(b)

(c) 
Fig. 15 Second specimen: dimensions in $\mathrm{mm}$

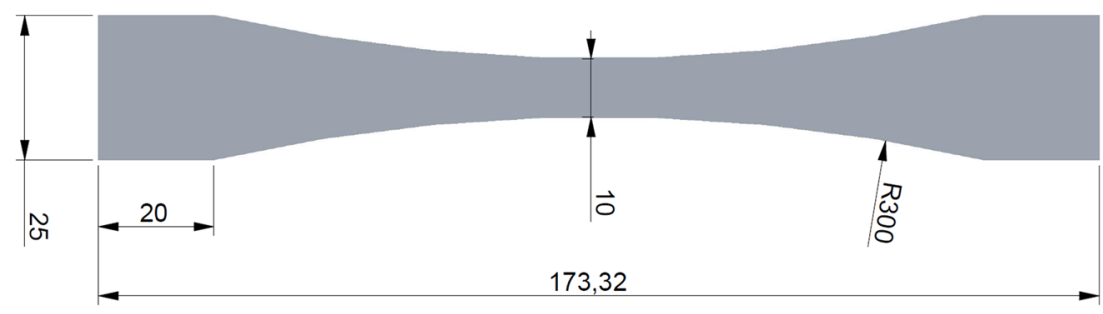

unloading cycles with an increasing maximum load level. Each cycle starts with a ten seconds pause at a null load level to acquire images of the unloaded sample and the jack speed is set at $0.1 \mathrm{~mm} . \mathrm{s}^{-1}$ during loading and at $0.02 \mathrm{~mm} . \mathrm{s}^{-1}$ during unloading. The image acquisition frequency is set at $5 \mathrm{~Hz}$. Note that the identification is performed here without using data associated with unloading steps.

Figure 17 illustrates the reference curve obtained using a classical dog-bone specimen and identifying the elastoplastic behavior on the stress-strain macroscopic response obtained using DIC. The "gauge length" of the strain measurement was $110 \mathrm{~mm}$.

\section{Identification Result}

The sample specimen is designed to generate controlled strain localization due to the stress gradient along the specimen axis. The loading and time steps for the identification are shown in Figure 18.

The meshes used for the identification are shown in Figure 19. Two multilinear elastic-plastic identifications are performed, i.e. the first one on a single material domain (Figure 19a) and the second on a ten material domains (Figure 19b). The DIC mesh consists in 640 quadrangles. The meshes for the elastic and plastic identifications respectively consist in 40 and 160 elements.

\section{Identification on one Material Domain}

For the first identification, the identified elastic constant values are equal to $173 \mathrm{GPa}$ for the Young's modulus and 0.18 for the
Poisson ratio. The identified yield strength $(355 \mathrm{MPa})$ is in agreement with the reference value (see Figure 20).

Five hardenings are identified and the identified elastoplastic parameters are also in good agreement with the reference values. The identified stress-strain curve is very similar to the reference curve (error less than 3\% in the hardening regime). Let us mention that the identified curve is reconstructed for a uniaxial tensile test using the identified parameters (yield stress, hardening moduli, ...). This curve is obtained assuming there is a single material domain. It does not correspond to the response of a specific location on the sample.

Figure 21 shows the identified stress distributions for the last plastic step. The fields are heterogeneous and the gradients are consistent with the expected results, as depicted by the different symmetries: the $\underline{\sigma_{x x}^{c}}$ and $\underline{\sigma_{y y}^{c}}$ stress fields are symmetric with respect to the axes $(O, x) \overline{\text { and }}(O, y)$ and the $\sigma_{x y}^{c}$ stress field has a central symmetry with respect to the specimen center $\mathrm{O}$. The shapes of these 'experimental' stress fields are very similar to those given by direct numerical simulation, and the amplitudes of the stress components are, by construction, compatible with the sample overall equilibrium. This confirms the validity of the identification method.

\section{Identification in ten Material Domains}

For the second identification, we perform elastic identification on a single material domain due to the poor signal-to-noise ratio in the elastic strain fields. The identified elastic constants are identical to those identified in the homogeneous case: $173 \mathrm{GPa}$ for the Young's modulus and 0.18 for the Poisson ratio.
Fig. 16 Overall strength (a) and overall displacement imposed on the cyclic test (b) (a)

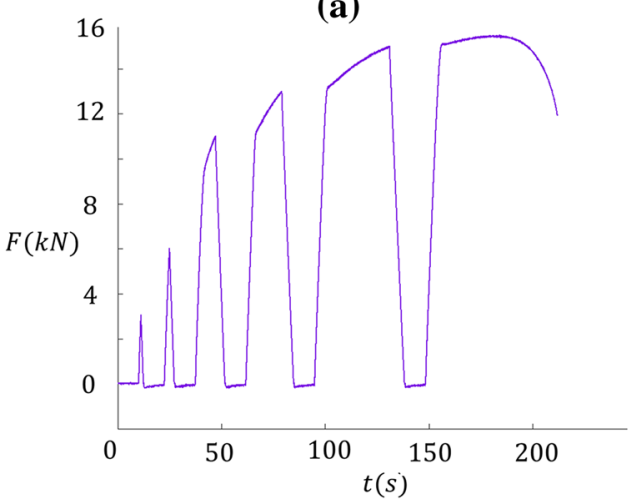

(b)

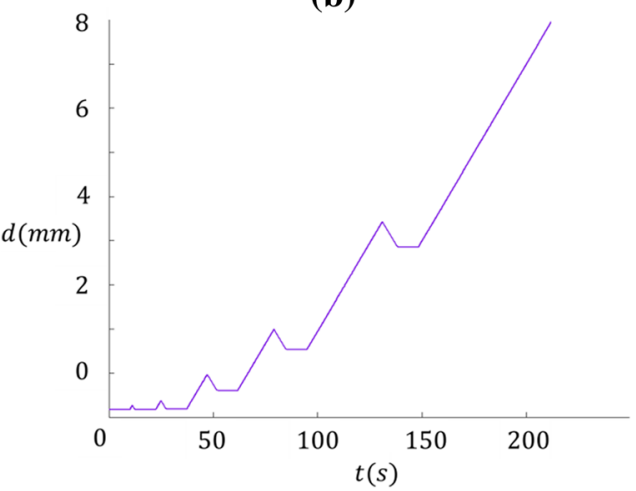


Fig. 17 Reference stress-strain curve for a simple traction test on DP600 steel

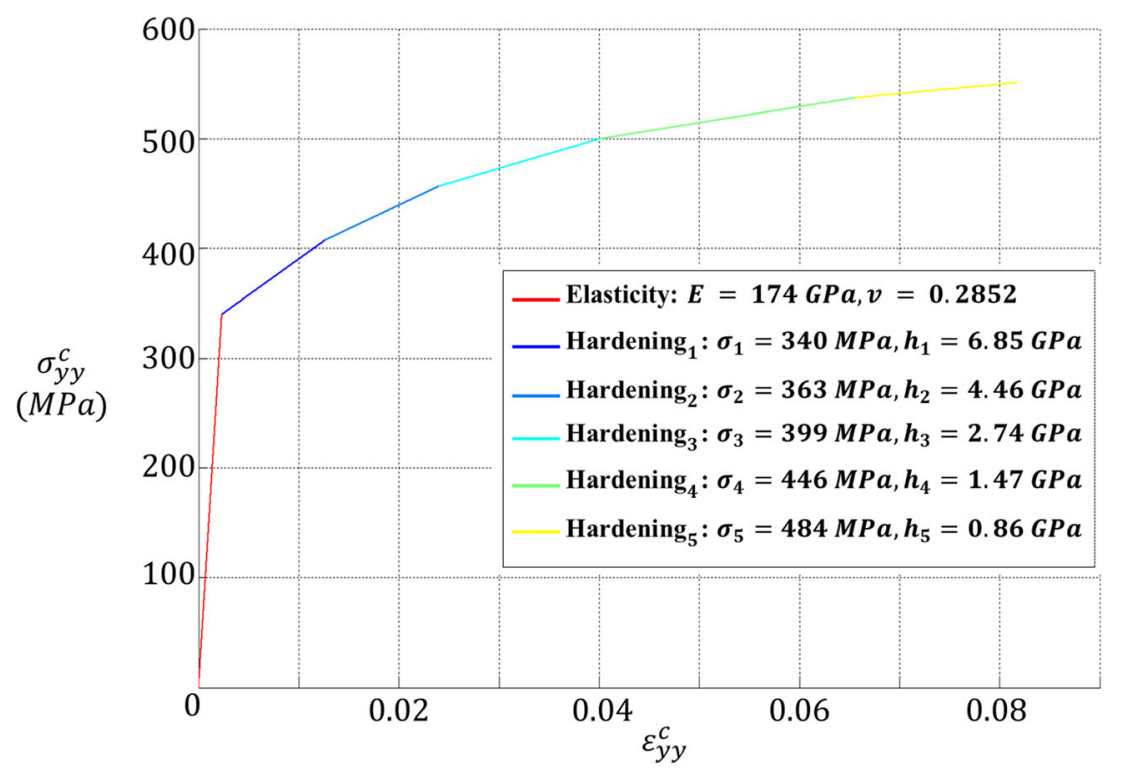

Figure 22 compares the plastic stress-strain curve for the first and second identification and the reference response. The hardening curves identified on each domain are plotted in the "equivalent plastic strain-von Mises equivalent stress" diagram using the identified parameters. Here again, these curves do not correspond to the response of a specific location on the sample. The material responses are also reconstructed from the parameters identified on each material domain. We mean that the responses identified on each material domain are very close to the one identified on a single domain (on the same specimen) and also to the one determined on the standard dog-bone specimen.

The ten responses indicate the symmetry in the identification results compatible with the stress and strain gradients imposed by the sample geometry. The differences in the ten material responses are due to the fact that the identification has been performed using a very limited set of three load steps (each one corresponding

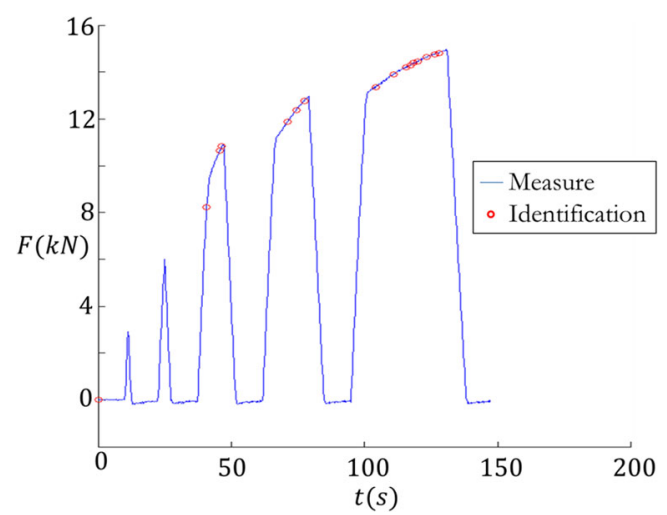

Fig. 18 Global load measured during the test and load steps used for identification to one recorded image). For a given macroscopic load step, the mechanical states of the material domains are different due to the stress and strain gradients along the sample axis: the center of the specimen being more strained than the ends of the specimen. When the sample is divided into ten material domains, the gradients within each material domain are very limited.

The space covered by the mechanical state (von Mises stress vs plastic strain) and used in the identification is discrete. When the mechanical fields are homogeneous on a given material domain for a given image (or applied load), a single material state is obtained. When the mechanical fields are heterogeneous on the material domain, several material states are obtained on a single image. Naturally, the higher the gradients in the mechanical fields, the larger the set of material states. In the situation presented in the paper, when the sample is divided into ten material domains, the mechanical gradients are very limited on each domain. Consequently, using the limited set of images, it is not possible to perfectly describe the whole material states and the identified properties are slightly different than the "reference one". Increasing the number of images used in the identification would make this differences vanish.

Finally, this result confirmed the homogeneity of the tested material and demonstrated the ability of the method to identify local material responses. Furthermore, the two identification results were in good agreement with the reference response identified in the same material but on a standard tensile specimen.

The identified stress distributions are realistic and consistent with those given by the first identification (see Figure 23). In this experiment, the heterogeneous stress field along the sample axis is correctly identified. 
Fig. 19 Identification in one (a) and ten material domains (b), mesh for elastic identification (c), for plastic identification (d) and for correlation (e)

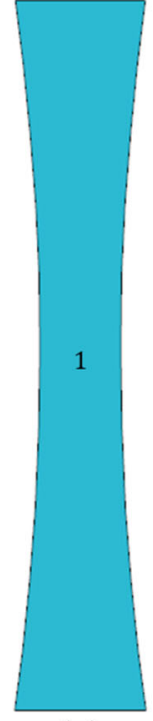

(a)

\section{Conclusions and Prospects}

In this study, a constitutive equation gap method was proposed to identify heterogeneous multilinear elastoplastic behaviors. The numerical formulation involved several regularization levels. The first one consisted in the a priori description of the material heterogeneity through the definition of the material mesh. The second one was obtained by differentiating the spatial description of the identified stress gradients and the measured displacement gradients using suitable displacement and stress meshes. As the signal-to-noise ratio was different in elasticity and plasticity, we proposed to adapt the spatial description to the mechanical regime. The proposed method involved a cost function based on a variational approach that was separately convex.

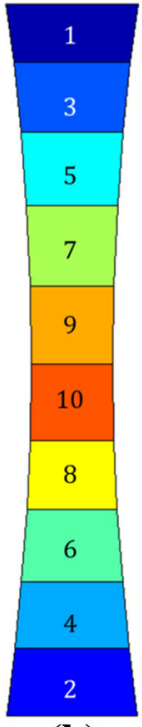

(b)

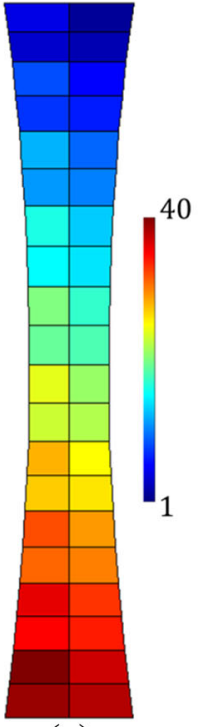

(c)

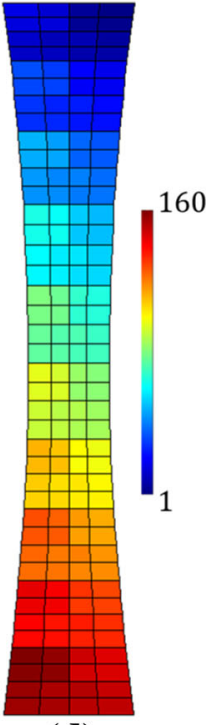

(d)

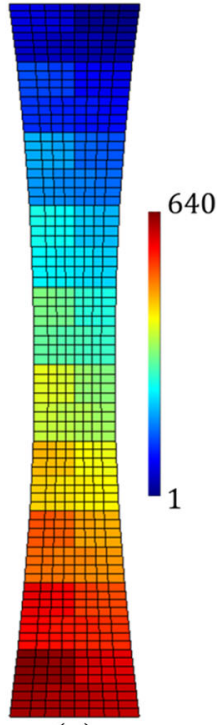

(e)
This last property still ensured the existence of a minimum and justified the use of a relaxation method for minimization of the functional. In a first step, the elastic properties were calculated explicitly by formulating the stationary of the cost function with respect to isotropic or cubic elastic coefficients. Plasticity onset detection allowed determination of the first plastic load step and to assign the elastic constants. The plastic identification was then performed. The main difficulty of this latter step concerned the local and simultaneous identification of plastic parameters using the stationarity of the constitutive equation gap functional with respect to the secant elastoplastic tensor parameters.

The identification process required knowledge of the geometry, of the different meshes (used to describe the measured displacements, the stress fields, and material properties), of the
Fig. 20 Superposition of the stress-strain curve identified in the specimen and macroscopic reference curve

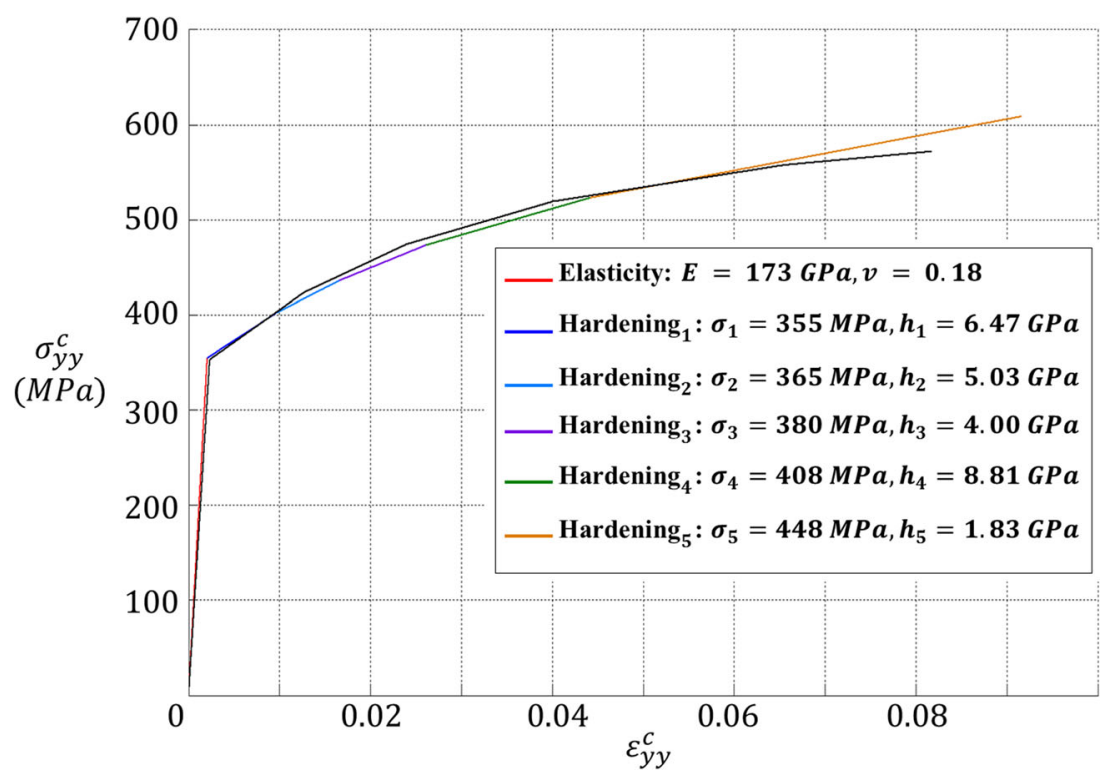




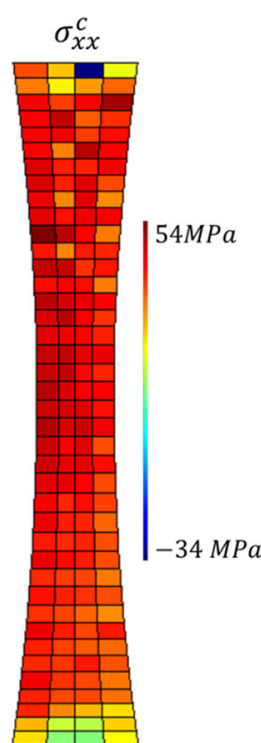

(a)

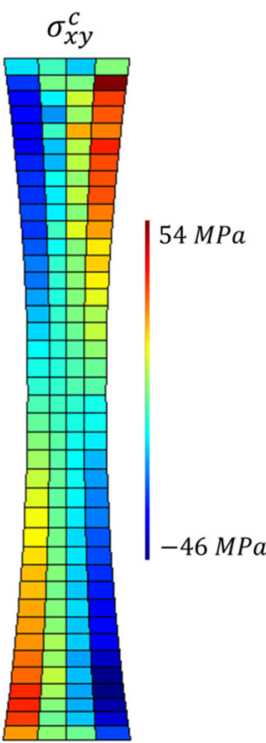

(b)

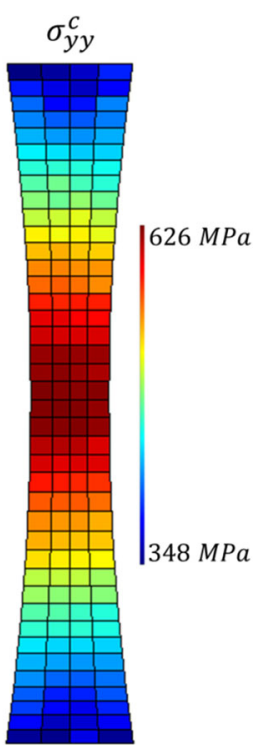

(c)

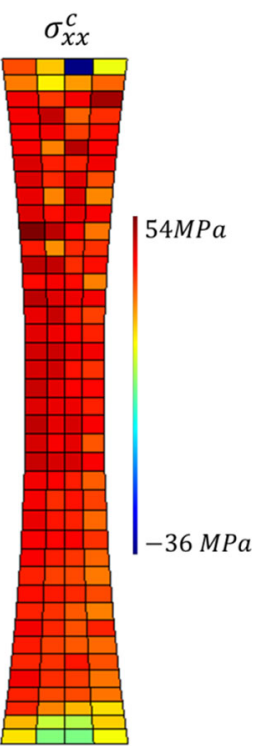

(a)

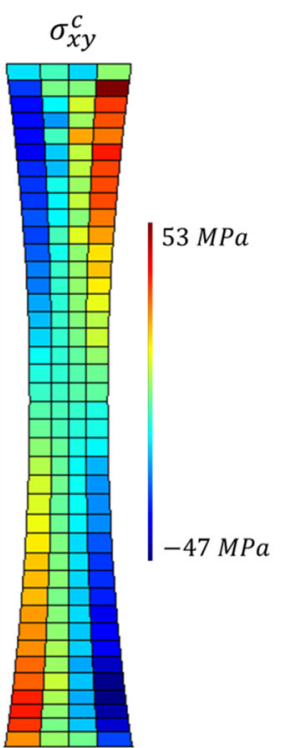

(b)

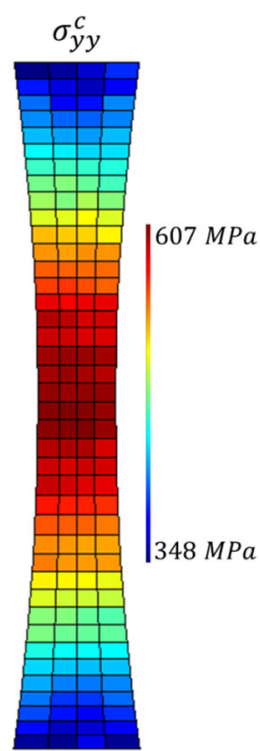

(c)

Fig. 21 Stress fields $\underline{\sigma}^{\mathrm{c}}$ identified in the longitudinal (a), shear (b) and transverse (c) directions for the last plastic loading step

measured displacement fields, of the free surface locations, and of the overall load levels on the loaded boundaries. All of these quantities were assumed to be known. In addition, an initial set of elastic parameters had to be introduced to initialize the identification procedure. The identification procedure did not require introduction of relevant initial values for identification of the plastic properties. They were obtained directly by secant elastic identification. This algorithm was used for plane problems for which the plane stress hypothesis held true.

In order to validate the identification protocol, several numerical examples providing access to heterogeneous deformation fields were proposed and the errors on the identified elastoplastic parameter and on the stresses were quantified.

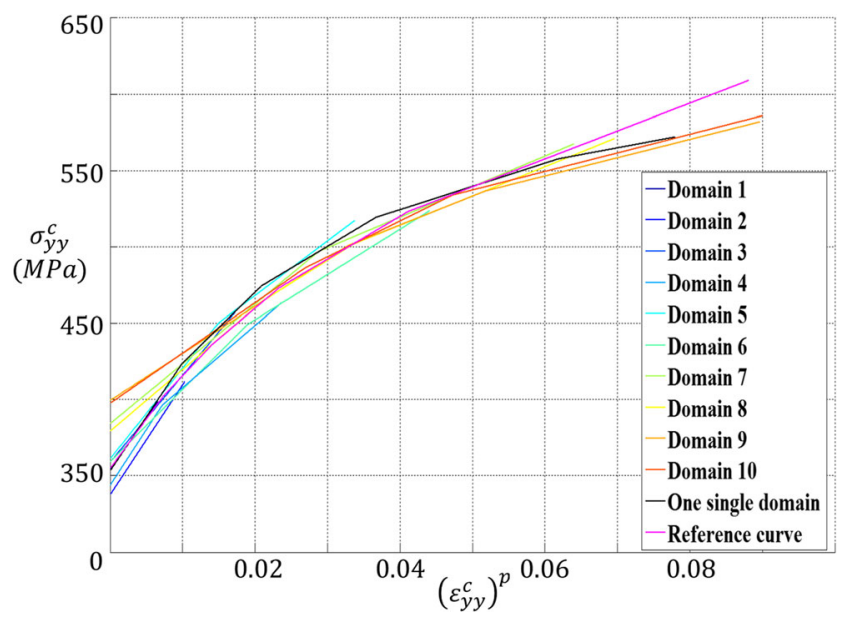

Fig. 22 Superposition of the plastic stress-strain curve identified one domain material (black), the reference curve (magenta) and in the ten domain materials
Fig. 23 Stress fields $\underline{\sigma}^{\mathrm{c}}$ identified in the longitudinal (a), shear (b) and transverse (c) directions for the last plastic loading step

The deformation fields were obtained by derivation of the numerically-obtained displacement fields potentially perturbed by Gaussian white noise. The use of several meshes for the elastic and plastic identification made it possible to filter the noise by projecting the displacements on coarser meshes chosen to describe the stress gradients.

These numerical tests allowed validation of the method on several points. The first was the ability of the method to reliably identify heterogeneous fields. The convergence stability of the method with respect to the size of the mesh and the choice of initialization parameters of the iterative procedure was demonstrated. Finally, the robustness of the inverse method with respect to measurement noise was evaluated and validated by the addition of Gaussian white noise.

Thereafter, the constitutive equation gap method was applied on the resulting kinematic fields measured during tensile tests on homogeneous specimens machined in the same batch of material (DP600 steel). The sample geometry was chosen to induce a controlled axial stress gradient. The identification was performed supposing a homogeneous or heterogeneous material. The identified parameters and the identified stress fields using these two hypotheses were consistent and in accordance with the homogeneity of the tested material. The identification results were also consistent with the material properties identified in a standard tensile geometry. As the proposed methodology was validated in slightly heterogeneous situations, we are now applying it to more heterogeneous situations such as crystal plasticity on aluminum multi-crystals. In this situation, the identification procedure could be further regularized by correlating the material properties with the crystal orientation measured by EBSD. 


\section{References}

1. Wattrisse B, Chrysochoos A, Muracciole J-M, Nemoz-Gaillard M (2001) Analysis of strain localization during tensile tests by digital image correlation. Exp Mech 41:29-39

2. Grédiac M (2004) The use of full-field measurement methods in composite material characterization: interest and limitations. Composites Part A: Appl Sci Manufac 35:751-761

3. Chalal H, Avril S, Pierron F, Meraghni F (2006) Experimental identification of a nonlinear model for composites using the grid technique coupled to the virtual fields method. Composites Part A: Appl Sci Manufac 37(2):315-325

4. Avril S, Pierron F (2007) General framework for the identification of constitutive parameters from full-field measurements in linear elasticity. Int J Solids Struct 44(14-15):4978-5002

5. Rossi M, Pierron F (2012) On the use of simulated experiments in designing tests for material characterization from full-field measurements. Int J Solids Struct 49(3-4):420-435

6. Avril S, Bonnet M, Bretelle A-S, Grédiac M, Hild F, Ienny P, Latourte F, Lemosse D, Pagano S, Pagnacco E, Pierron F (2008) Overview of identification methods of mechanical parameters based on full-field measurements. Experiment Mech 48(4):381-402

7. Kavanagh KT, Clough RW (1971) Finite element applications in the characterization of elastic solids. Int J Solids Struct 7:11-23

8. Meuwissen MHH, Oomens CWJ, Baaijens FPT, Petterson R, Janssen JD (1998) Determination of the elasto-plastic properties of aluminum using a mixed numerical-experimental method. J Mater Process Technol 75(1-3):204211

9. Cooreman S, Lecompte D, Sol H, Vantomme J, Debruyne D (2008) Identification of mechanical material behavior through inverse modeling and DIC. Exp Mech 48(4):421-433

10. Passieux J-C, Bugarin F., David C., Périé J-N and Robert L., Multiscale displacement field measurementusing digital image correlation: application to the identification of elastic properties. Experiment Mech, volume: 55, Issue: 1, Pages: 121-137, 2015

11. Mathieu F., Leclerc H., Hild F. and Roux S., Estimation of elastoplastic parameters via weighted FEMU and integrated-DIC. Experiment Mech, volume: 55, Issue: 1, Pages: 105-119, 2015

12. Guery A, Hild F, Latourte F, Roux S (2016) Identification of crystal plasticity parameters using DIC measurements and weighted FEMU. Mech Mater 100:55-71

13. Siddiqui M, Khan SZ, Khan MA, ShahzadK M, Khan KA, Nisar S, Noman D (2017) A projected finite element update method for inverse identification of material constitutive parameters in transversely isotropic laminates. Experiment Mech 57(5):755-772

14. Bui HD, Constantinescu A, Maigre H (2004) Numerical identification of linear cracks in 2D elastodynamics using the instantaneous reciprocity gap. Inverse Problems 20:993-1001

15. Sun Y, Guo Y, Ma F (2016) The reciprocity gap functional method for the inverse scattering problem for cavities. Appl Anal 95(6): $1327-1346$

16. Feissel P, Allix O (2007) Modified constitutive relation error identification strategy for transient dynamics with corrupted data: the elastic case. Comput Eng Appl Mech Eng 196:1968-1983

17. Florentin E, Lubineau G (2011) Using constitutive equation gap method for identification of elastic material parameters: technical insights and illustrations. Int J Interact Design Manufac (IJIDeM) 5(4):227-234
18. Merzouki T, Nouri H, Roger F (2014) Direct identification of nonlinear damage behavior of composite materials using the constitutive equation gap method. Int J Mech Sci 89:487-499

19. Grédiac M, Toussaint E, Pierron F (2002) Special virtual fields for the direct determination of material parameters with the virtual fields method.1 - Principle and definition. Int J Solids Struct 39: 2691-2705

20. Grédiac M, Pierron F (2006) Applying the virtual field method to the identification of elasto-plastic constitutive parameters. Int J Plast 22:602-627

21. Kim J-H, Pierron F, Wisnom MR, Syed-Muhamad K (2007) Identification of the local stiffness reduction of a damaged composite plate using the virtual fields method. Composites Part A: Appl Sci Manufac 38(9):2065-2075

22. Avril S, Huntley JM, Pierron F, Steele DD (2008) 3D heterogeneous stiffness reconstruction using MRI and the virtual fields method. Exp Mech 48(4):479-494

23. Pierron F, Avril S, The TV (2010) Extension of the virtual fields method to elastoplastic material identification with cyclic loads and kinematic hardening. Int J Solids Struct 47(22-23):2993-3010

24. Grama SN, Subramanian SJ, Pierron F (2015) On the identifiability of Anand visco-plastic model parameters using the virtual fields method. Acta Mater 86:118-136

25. Rossi M, Pierron F, Štamborská M (2016) Application of the virtual fields method to large strain anisotropic plasticity. Int J Solids Struct 97-98:322-335

26. Wang P, Pierron F, Rossi M, Lava P, Thomsen OT (2016) Optimised experimental characterisation of polymeric foam material using DIC and the virtual fields method. Strain 52(1):59-79

27. Marek A, Davis FM, Pierron F (2017) Sensitivity-based virtual fields for computational mechanics. Comput Mech 60(3):409-431

28. Roux S, Hild F, Pagano S (2005) A stress scale in full-field identification procedures: a diffuse stress gauge. Eur J Mech - A/Solids 24:442451

29. Ben AM, Périé J-N, Guimard J-M, Hild F, Roux S (2011) On the identification and validation of an anisotropic damage model using full-field measurements. Int J Damage Mech 20(8):1130-1150

30. Ladevèze P (1983) And and Leguillon D., Error estimate procedure in the finite element method and applications. SIAM. J Num Anal 20:485-509

31. Constantinescu A (1995) On the identification of elastic modulus from displacement force boundary measurements. Inverse Problems Eng 1:293-315

32. Geymonat G, Pagano S (2003) Identification of mechanical properties by displacement field measurement: a variational approach. Meccanica 38:535-545

33. Latourte F, Chrysochoos A, Pagano S, Wattrisse B (2008) Elastoplastic behavior identification for heterogeneous loadings and materials. Exp Mech 48:435-449

34. Simo J, Hughes T (1998) Computational inelasticity. Springer, Verlag

35. Bornert M, Brémand F, Doumalin P, Dupré M, Fazzini J-C, Grédiac M, Hild F, Mistou S, Molimard J, Orteu J-J, Robert L, Surrel Y, Vacher P, Wattrisse B (2008) Assessment of digital image correlation measurement errors: methodology and results. Exp Mech 49: 353-370 